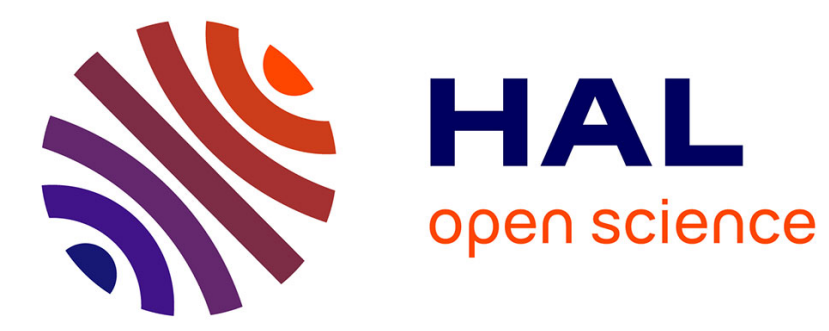

\title{
Etude comparative du couplage endommagement-fluage
}

Mirvat Omar, Gilles Pijaudier-Cabot, Ahmed Loukili

\section{To cite this version:}

Mirvat Omar, Gilles Pijaudier-Cabot, Ahmed Loukili. Etude comparative du couplage endommagement-fluage. Revue Française de Génie Civil , 2004, 8 (4), pp.457 - 481. 10.1080/12795119.2004.9692615 . hal-01006809

\section{HAL Id: hal-01006809 \\ https://hal.science/hal-01006809}

Submitted on 3 Feb 2017

HAL is a multi-disciplinary open access archive for the deposit and dissemination of scientific research documents, whether they are published or not. The documents may come from teaching and research institutions in France or abroad, or from public or private research centers.
L'archive ouverte pluridisciplinaire $\mathbf{H A L}$, est destinée au dépôt et à la diffusion de documents scientifiques de niveau recherche, publiés ou non, émanant des établissements d'enseignement et de recherche français ou étrangers, des laboratoires publics ou privés.

\section{(c)(1)}

Distributed under a Creative Commons Attribution| 4.0 International License 


\title{
Etude comparative du couplage endommagement-fluage
}

\section{Mirvat Omar — Gilles Pijaudier-Cabot — Ahmed Loukili}

\author{
Ecole Centrale de Nantes - Université de Nantes - CNRS \\ $R \& D O-G e M-$ Institut de Recherche en Génie Civil et Mécanique \\ 1, rue de la Noё BP 92101 \\ F-44321 Nantes cedex 3
}

RÉSUMÉ. Ce travail a pour objectif d'aboutir à une modélisation numérique robuste du fluage et de son interaction avec les propriétés de fissuration du béton. Deux méthodes de couplage entre l'endommagement non local et le fluage sont présentées. Dans la première méthode, le fluage est modélisé à l'aide du modèle de Maxwell et l'endommagement apparaît à partir du concept de contraintes effectives. Dans la seconde, la modélisation du fluage est basée sur le modèle de Kelvin où l'endommagement agit sur la partie indépendante du temps dans la fonctionnelle de fluage. Les paramètres des modèles numériques ont été identifiés sur des données expérimentales. Des simulations numériques de fluage et de relaxation sont présentées. Les résultats des différents programmes de sollicitations avec différents paramètres de contrôle confirment l'influence du comportement endommageable du béton sur le fluage ou la relaxation. La cinétique de fluage des poutres en flexion est fortement influencée par la longueur interne du matériau lorsque l'endommagement a lieu.

ABSTRACT: The aim of this research is to investigate numerical modeling of coupling between creep and crack growth in concrete. The paper underlines the different approaches that can be envisioned in order to incorporate damage into the time dependent creep functions: In the Maxwell model, the effective stress approach was used. In the Kelvin model, material damage acts on the time independent part of the creep functional. The numerical models are calibrated so that they fit the experimental data. Some computer simulations have been carried out. Results of various loading histories with different controlling parameters confirm the influence of the damage behaviour on creep or relaxation of concrete. Actually, the kinetics of creep in bending beams is strongly correlated with the internal length when damage occurs.

MoTS-CLÉs: endommagement, béton, calcul éléments finis non linéaire, fluage propre, relaxation.

KEYWORDS: damage, concrete, non linear finite element computations, basic creep, relaxation. 


\section{Introduction}

La viscoélasticité et la croissance des fissures gouvernent la déformabilité à long terme du béton et donc son comportement en service et sa durabilité. Pour des niveaux de sollicitation bas, la viscoélasticité peut être considérée comme linéaire et le comportement mécanique instantané du matériau est élastique. En revanche, pour des niveaux de sollicitation élevés, la microfissuration se développe et interagit avec la viscoélasticité. L'influence du fluage sur le comportement instantané du béton a été mise en évidence de manière qualitative par de nombreux auteurs. Les premiers résultats expérimentaux remontent à (Rüsch, 1958). Ainsi, la résistance mécanique d'une éprouvette, préalablement sollicitée en fluage, diminue avec la durée d'application de la sollicitation initiale. Gettu et Bazant ont aussi montré l'influence de la vitesse de chargement sur la résistance à la fissuration et son effet sur les diagrammes d'effet d'échelle (Gettu, 1992), (Bazant, 1998). Leur étude portait sur des vitesses de chargement variant entre $4 \times 10^{-11} \mathrm{~m} / \mathrm{s}$ et $10^{-5} \mathrm{~m} / \mathrm{s}$, elle a montré que plus la vitesse de chargement est lente plus les éprouvettes deviennent fragiles. Ainsi la taille de la zone de microfissuration entourant une macrofissure, se propageant en mode I, diminue avec la vitesse de chargement. Des modèles de fissuration discrète où les effets visqueux apparaissent existent aussi. Santhikumar et Karihaloo (Santhikumar, 1996) et $\mathrm{Zi}$ et Bazant ( $\mathrm{Zi}, 2001)$ ont proposé de telles formulations. Celles-ci ne permettent cependant pas de traiter les cas ou la microfissuration est diffuse.

La modélisation des effets mécaniques couplés entre le comportement viscoélastique du matériau, vu comme un milieu continu, et son comportement non linéaire instantané, a souvent été abordée en utilisant un modèle de plasticité placé en série avec un modèle de fluage linéaire. De Borst en a fait une synthèse en 1993 (De Borst, 1993). Notons d'ailleurs que le comportement instantané du matériau peut être décrit indifféremment par un modèle de plasticité, un modèle de fissuration diffuse (Rots, 1988) ou un modèle d'endommagement (Omar, 2000).

Pourtant ce type de couplage n'est pas le seul possible. Dans cette contribution, nous comparons deux méthodes de couplage possibles entre le comportement endommageable du béton et son comportement visqueux. Deux modèles de comportement viscoélastiques distincts (le modèle rhéologique de la chaîne de Kelvin et le modèle rhéologique de la chaîne de Maxwell (Bazant, 1974) sont envisagés. Le premier est couplé à un modèle d'endommagement en série et le second est couplé à l'endommagement par l'intermédiaire de la contrainte effective, ce qui est une approche très classique utilisée pour les métaux (Lemaitre et Chaboche, 1984). Nous envisageons donc deux techniques de couplage très distinctes à partir de modèles mécaniques similaires, afin d'en apprécier les qualités et les défauts. Pour simplifier cette comparaison, nous emploierons des modèles unidimensionnels implantés dans un code de calcul d'ossatures sollicitées en traction et en flexion. 
Ces deux modèles sont tout d'abord décrits et leur implémentation dans un code de calcul par éléments finis est traitée. Des simulations numériques de fluage et de relaxation en traction et en flexion sont présentées. Divers programmes de sollicitation, avec différents paramètres de contrôle, permettent d'analyser la sensibilité du comportement instantané sur le comportement différé ainsi que les interactions existantes entre viscoélasticité et endommagement, selon les modes de couplage.

\section{Lois de comportement}

Dans cette contribution, nous employons une formulation simplifiée du comportement viscoélastique du béton, car nous recherchons seulement à exhiber les caractéristiques du couplage entre l'endommagement et le fluage. Nous employons un modèle linéaire classique. Dans le régime viscoélastique, la déformation $\varepsilon(t)$ à un instant $t$ est une fonction de l'histoire des contraintes $\sigma$, sous la forme d'une équation intégrale de Bolzmann :

$$
\varepsilon(t)=J\left(t_{0}, t\right) \sigma\left(t_{0}\right)+\int_{t_{0}}^{t} J(\tau, t) \dot{\sigma}(\tau) d \tau
$$

où $\mathrm{J}\left(\mathrm{t}_{0}, \mathrm{t}\right)$ est la fonctionnelle de fluage. $\mathrm{t}_{0}$ est l'instant du début du chargement. $\mathrm{A}$ chaque instant $\dot{\sigma}(\tau)$ est la dérivée de la contrainte par rapport au temps. Cette équation peut être inversée pour être écrite sous la forme :

$$
\sigma(\mathrm{t})=\mathrm{R}\left(\mathrm{t}_{0}, \mathrm{t}\right) \varepsilon\left(\mathrm{t}_{0}\right)+\int_{\mathrm{t}_{0}}^{\mathrm{t}} \mathrm{R}(\tau, \mathrm{t}) \dot{\varepsilon}(\tau) \mathrm{d} \tau
$$

où $\mathrm{R}\left(\mathrm{t}_{0}, \mathrm{t}\right)$ est la fonctionnelle de relaxation. Nous supposerons dans la suite que le matériau est non vieillissant, ce qui signifie que la réponse instantanée du matériau ne dépend pas de son âge. Ainsi, l'expression des fonctionnelles de fluage ou de relaxation sera simplifiée. L'équation [1] sera approximée par une chaîne de Kelvin et l'équation [2] sera approximée par une chaîne de Maxwell. Par souci de simplicité, le comportement viscoélastique du matériau sera considéré comme indépendant du signe de la contrainte. Le fluage en traction est identique au fluage en compression. A ce sujet, Bissonnette a mené une étude comparative des deux phénomènes (Bissonnette, 1995). Weiss (Weiss, 1999) et Østergaard (Østergaard, 2001) ont aussi souligné la similarité entre la fonctionnelle de fluage en traction et en compression.

Avant de discuter ces deux approches et leur couplage avec le comportement instantané, commençons par rappeler les principaux éléments du modèle de comportement instantané employé. Il s'agit du modèle d'endommagement scalaire proposé par Mazars (Mazars, 1984). Dans le contexte de calcul de structures de type 
poutre, ce dernier permet d'obtenir une représentation réaliste d'éléments de structure fléchis sous chargement monotone (Bazant, 1987ab). Afin d'éviter les problèmes théoriques et numériques liés au comportement adoucissant du matériau, nous employons une version non locale de ce modèle (Pijaudier-Cabot, 1987).

\subsection{Endommagement mécanique}

L'effet mécanique de la microfissuration due au chargement extérieur est décrit par une variable scalaire $d$. La relation contrainte déformation prend la forme :

$$
\sigma=(1-\mathrm{d}) \mathrm{E} \varepsilon
$$

E est le module d'Young initial du matériau. Pour décrire la variation de l'endommagement on définit tout d'abord la déformation équivalente $\widetilde{\varepsilon}$. Dans ce modèle unidimensionnel, nous avons simplement :

$$
\tilde{\varepsilon}=\langle\varepsilon\rangle_{+}
$$

$\langle\varepsilon\rangle_{+}=0 \quad$ si $\quad \varepsilon<0$ et $\langle\varepsilon\rangle_{+}=\varepsilon^{\mathrm{i}} \quad$ si $\quad \varepsilon \geq 0$. Dans l'approche non locale, la variable contrôlant l'endommagement est la moyenne de cette déformation effective :

$$
\bar{\varepsilon}(\mathrm{x})=\frac{1}{\mathrm{~V}_{\mathrm{r}}(\mathrm{x})} \int_{\Omega} \psi(\mathrm{x}-\mathrm{s}) \widetilde{\varepsilon}(\mathrm{s}) \mathrm{ds} \text { avec } \mathrm{V}_{\mathrm{r}}(\mathrm{x})=\int_{\Omega} \psi(\mathrm{x}-\mathrm{s}) \mathrm{ds}
$$

où $\Omega$ est le volume de la structure, $\operatorname{Vr}(\mathrm{x})$ est le volume représentatif au point $x$, et $\psi(x-s)$ est la fonction de pondération non locale :

$$
\psi(\mathrm{x}-\mathrm{s})=\exp \left(-\frac{4\|\mathrm{x}-\mathrm{s}\|^{2}}{\mathrm{l}_{\mathrm{C}}^{2}}\right)
$$

$l_{c}$ est la longueur interne. Notons que dans le cas d'une barre ou d'une poutre, les intégrales sont des intégrales simples et le volume représentatif est unidimensionnel. $\mathrm{La}$ déformation moyenne (équation [5]) contrôle l'accroissement de l'endommagement sous les conditions suivantes :

$$
\mathrm{F}(\bar{\varepsilon})=\bar{\varepsilon}-\kappa
$$

si F $(\bar{\varepsilon})=0$ et $\dot{\mathrm{F}}(\bar{\varepsilon})=0$ alors $\left\{\begin{array}{l}\dot{\mathrm{d}}=\mathrm{h}(\kappa) \\ \dot{\mathrm{\kappa}}=\dot{\bar{\varepsilon}}\end{array}\right.$ avec $\dot{\mathrm{d}} \geq 0$ sinon $\left\{\begin{array}{l}\dot{\mathrm{d}}=0 \\ \dot{\mathrm{\kappa}}=0\end{array}\right.$

La surface seuil est définie par $\mathrm{F}(\bar{\varepsilon})=0$ et $\dot{\mathrm{d}} \geq 0$. Dans la loi d'évolution $h(\kappa)$, l'endommagement est usuellement donné sous forme intégrée en temps et décomposé en deux parties, $d_{t}$ pour la traction et $d_{c}$ pour la compression. Dans notre 
cas, seul l'endommagement en traction sera considéré $\left(\mathrm{d}_{\mathrm{c}}=0\right)$. Il suit la loi d'évolution :

$$
\mathrm{d}_{\mathrm{t}}=1-\frac{\kappa_{0}\left(1-\mathrm{A}_{\mathrm{t}}\right)}{\kappa}-\frac{\mathrm{A}_{\mathrm{t}}}{\exp \left(\mathrm{B}_{\mathrm{t}}\left(\kappa-\kappa_{0}\right)\right)}
$$

$A_{t}, B_{t}$ sont les paramètres du modèle. Pour des bétons classiques :

$$
0.7 \leq \mathrm{A}_{\mathrm{t}} \leq 1.2,10^{4} \leq \mathrm{B}_{\mathrm{t}} \leq 5.10^{4}
$$

\subsection{Chaîne de Kelvin couplée à l'endommagement}

La fonctionnelle de fluage (équation [1]) est développée en série de Dirichlet (Bazant, 1973). Nous obtenons alors l'analogue d'une chaîne de Kelvin :

$$
J(\tau, t)=\frac{1}{E_{0}}+\sum_{\mu=1}^{n} \frac{1}{E_{\mu}}\left[1-\exp \left(-\frac{t-\tau}{\tau_{\mu}}\right)\right]
$$

où $\tau_{\mu}$ et $\mathrm{E}_{\mu}$ sont les temps de relaxation et les modules de comportement instantanné de chaque élément de Kelvin. $n$ représente le nombre d'éléments de Kelvin placés en série comme indiqué sur la figure 1.

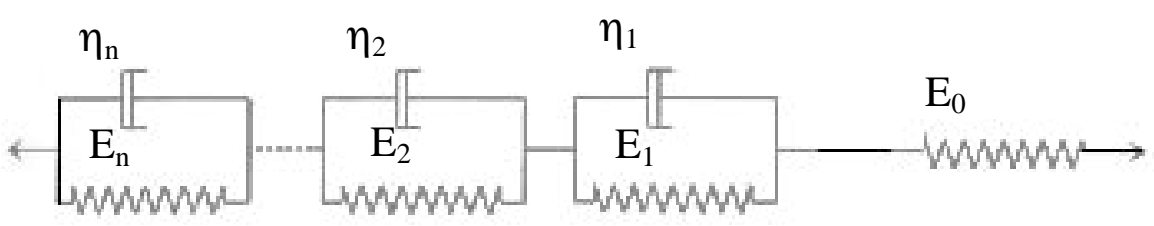

Figure 1. Schéma rhéologique correspondant à une chaîne de Kelvin

Ce type de loi de comportement viscoélastique a été utilisé en particulier par Granger (Granger, 1995). En substituant cette expression dans l'équation [1] nous obtenons la relation :

$$
\varepsilon(\mathrm{t})=\int_{\tau=\mathrm{t}_{0}}^{\tau=\mathrm{t}} \frac{\dot{\sigma}(\tau)}{\mathrm{E}_{0}} \mathrm{~d} \tau+\int_{\tau=\mathrm{t}_{0}}^{\tau=\mathrm{t}} \sum_{\mu=1}^{\mathrm{n}} \frac{1}{\mathrm{E}_{\mu}}\left[1-\exp \left(\frac{\mathrm{t}-\tau}{\tau_{\mu}}\right)\right] \dot{\sigma}(\tau) \mathrm{d} \tau
$$

Dans cette équation, nous pouvons alors séparer la réponse instantanée en déformation $\varepsilon^{\text {in }}(\mathrm{t})$ de la réponse différée $\varepsilon^{\mathrm{fl}}(\mathrm{t})$ (c'est-à-dire dépendant du temps). Ainsi :

$$
\varepsilon(t)=\varepsilon^{i n}(t)+\varepsilon^{f l}(t)
$$


avec :

$$
\varepsilon^{\mathrm{in}}(\mathrm{t})=\int_{\tau=\mathrm{t} 0}^{\tau=\mathrm{t}} \frac{\dot{\sigma}(\tau)}{\mathrm{E}_{0}} \mathrm{~d} \tau \quad \text { et } \quad \varepsilon^{\mathrm{fl}}(\mathrm{t})=\int_{\tau=\mathrm{t} 0}^{\tau=\mathrm{t}} \sum_{\mu=1}^{\mathrm{n}} \frac{1}{\mathrm{E}_{\mu}}\left[1-\exp \left(\frac{\mathrm{t}-\tau}{\tau_{\mu}}\right)\right] \dot{\sigma}(\tau) \mathrm{d} \tau
$$

En s'inspirant de la méthode proposée par De Borst (De Borst, 1993), et appliquée parmi d'autres par Witasse (Witasse, 2000), nous considérons alors que l'endommagement agit sur la partie instantanée du comportement seulement. La déformation instantanée devient :

$$
\varepsilon^{\text {in }}(t)=\frac{\sigma(t)}{E(1-d)}
$$

où l'endommagement $d$ est évalué en remplaçant la déformation par la déformation instantanée $\varepsilon^{\text {in }}(\mathrm{t})$ dans les équations contrôlant la croissance de l'endommagement (de l'équation [4] à l'équation [9]). Cette forme de couplage suppose donc que l'endommagement affecte le ressort placé à droite sur la figure 1.

L'incrément $\Delta \varepsilon^{\mathrm{fl}}(\mathrm{t}) \mathrm{s}$ 'exprime sous la forme :

$$
\Delta \varepsilon^{\mathrm{fl}}(\mathrm{t})=\varepsilon^{\mathrm{fl}}(\mathrm{t}+\Delta \mathrm{t})-\varepsilon^{\mathrm{fl}}(\mathrm{t})=\sum_{\mathrm{q}=1}^{\mathrm{r}} \sum_{\mu=1}^{\mathrm{n}} \frac{1}{\mathrm{E}_{\mu} \tau_{\mu}}\left[\exp \left(\frac{\mathrm{r} \Delta \mathrm{t}-\mathrm{q} \Delta \tau}{\tau_{\mu}}\right)\right] \Delta \sigma_{\mathrm{q}} \Delta \mathrm{t}
$$

Nous supposons ici que les incréments de temps sont constants, $\mathrm{t}=\mathrm{t}_{0}+\mathrm{r} \Delta \mathrm{t}$ et $\tau=\mathrm{t}_{0}+\mathrm{q} \Delta \mathrm{t} . \Delta \sigma_{\mathrm{q}}$ est l'incrément de contrainte enregistré pendant chaque intervalle de temps : $\Delta \sigma_{\mathrm{q}}=\sigma\left(\mathrm{t}_{0}+\mathrm{q} \Delta \mathrm{t}\right)-\sigma(\mathrm{t} 0+(\mathrm{q}-1) \Delta \mathrm{t})$. Notons que le calcul de la déformation de fluage est complètement explicite. Ainsi, la déformation de fluage à l'instant $\mathrm{t}+\Delta \mathrm{t}$ ne dépend que de l'histoire de chargement entre $\mathrm{t}_{0}$ et $\mathrm{t}$ et du pas de temps supposé suffisamment petit.

Dans la plupart des applications pratiques, $\mathrm{n} \geq 8$. Cette intégration suppose que l'histoire du chargement soit conservée en mémoire à chaque pas. Cette difficulté, liée à une formulation héréditaire de type Kelvin, a été soulignée de nombreuses fois (voir par exemple l'ouvrage de synthèse (Bazant, 1988).

Le code de calculs EFICOS, que nous utilisons pour le calcul non linéaire de poutres, emploie un algorithme de résolution basé sur la méthode sécante (Bazant, 1987a). A chaque incrément de temps, nous résolvons l'équation :

$$
\mathrm{K}^{\mathrm{sec}} \overrightarrow{\mathrm{u}}=\overrightarrow{\mathrm{f}}
$$

$K^{\mathrm{sec}}$ est la matrice sécante de la structure, $\overrightarrow{\mathrm{u}}$ est le vecteur de déplacements nodaux et $\vec{f}$ celui des efforts extérieurs. La matrice de raideur sécante est calculée à partir de la raideur sécante de chaque élément poutre discrétisée en couches. Cette dernière est formée par assemblage des raideurs sécantes locales $E^{\text {sec }}$ de chaque couche : 


$$
\mathrm{E}^{\sec }(\mathrm{t})=\frac{\sigma(\mathrm{t})}{\varepsilon(\mathrm{t})} \text { et } \mathrm{K}^{\mathrm{sec}}=\int_{\mathrm{v}} \mathrm{B}^{\mathrm{T}} \mathrm{E}^{\mathrm{sec}} \mathrm{Bdv} \text { avec } \varepsilon=\mathrm{B} \overrightarrow{\mathrm{u}}
$$

Comme l'endommagement est une fonction explicite de la déformation instantanée et comme la déformation de fluage est connue à chaque pas de temps, cette raideur locale est elle-même explicitement connue à chaque pas de temps pour un champ de déplacement fixé (qui ne vérifie pas forcement l'équation [17]). La convergence est obtenue lorsque les forces résiduelles sont plus petites qu'une certaine tolérance. Cet algorithme présente l'avantage de ne pas avoir à exprimer la raideur tangente du matériau. Celle-ci en effet est non symétrique pour un modèle non local (Pijaudier-Cabot, 1991) et son emploi requiert l'implémentation d'un solveur plus complexe.

\subsection{Chaîne de Maxwell couplée à l'endommagement}

Dans ce paragraphe, nous considérons que la fonctionnelle de relaxation (équation [2]) est développée en série de Dirichlet (Bazant, 1974) :

$$
\mathrm{R}(\tau, \mathrm{t})=\mathrm{E}_{0}+\sum_{\mu=1}^{\mathrm{n}} \mathrm{E}_{\mu} \exp \left(-\tau_{\mu}(\mathrm{t}-\tau)\right)
$$

$\tau_{\mu}$ et $\mathrm{E}_{\mu}$ sont respectivement les temps de relaxation et les modules d'élasticité de chaque branche de la chaîne de Maxwell. Généralement, les valeurs de $\tau_{\mu}$ sont données a priori et chaque valeur de $\mathrm{E}_{\mu}$ est alors déterminée par une méthode des moindres carrés (Bazant, 1973, 1974). Usuellement, $\mathrm{n}=4$ pour une bonne représentation d'une fonctionnelle de fluage pour un béton classique. La figure 2 montre un schéma rhéologique d'une chaîne de Maxwell.

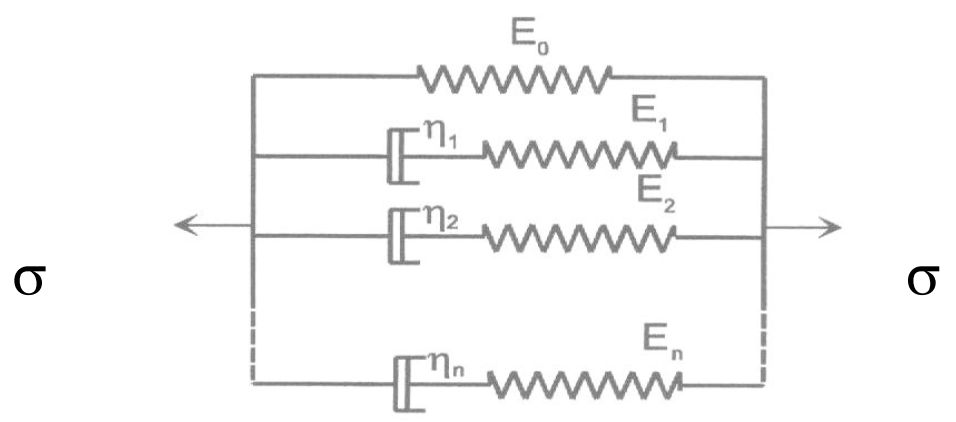

Figure 2. Schéma rhéologique de la chaîne de Maxwell

Avant de discuter du couplage avec l'endommagement, il est nécessaire de remarquer que les approches via une chaîne de Kelvin ou via une chaîne de Maxwell sont complètement équivalentes en termes de représentation du comportement 
viscoélastique du matériau. Tant que le modèle n'est pas couplé à un comportement non linéaire instantané, ou à un autre effet conduisant à un comportement mécanique non linéaire, ces deux modèles sont strictement équivalents. Il est possible de passer aisément de l'un à l'autre et la préférence pour l'une ou l'autre des deux formulations dépend essentiellement de leur facilité d'identification et d'implantation numérique.

Le couplage avec le comportement endommageable du matériau peut s'effectuer de diverses façons. Nous pouvons en effet considérer que l'endommagement affectera chaque branche de la chaîne de Maxwell de façon indépendante (en fonction de la déformation du ressort dans chacune d'entre elles), ou que celui-ci va, globalement influer sur le comportement résultant. C'est cette approche qui est retenue ici en utilisant le concept de contrainte effective. Celle-ci, notée $\sigma^{t}$ est définie comme étant la contrainte supportée par le matériau, une fois déduite l'aire des microfissures (Lemaitre, Chaboche, 1984). Elle est calculée en fonction de la contrainte macroscopique appliquée au matériau et en fonction de son état d'endommagement :

$$
\sigma^{\mathrm{t}}=\frac{\sigma}{(1-\mathrm{d})}
$$

La contrainte appliquée à la chaîne de Maxwell, décrite sur la figure 2, devient la contrainte effective. Nous supposons ainsi que le matériau, une fois les microfissures enlevées, a un comportement viscoélastique linéaire. Ce type de couplage, particulièrement simple est relativement classique pour les métaux à haute température. Son application au comportement du béton a été suggéré par Omar (Omar, 2000), Benboudjema (Benboudjema, 2001), et Mazotti (Mazzotti, 2001).

A partir des équations [2] et [20], il est possible de calculer l'incrément de contrainte effective $\Delta \sigma^{t}$ entre les pas de temps t et $t+\Delta t$ correspondant à un incrément de déformation $\Delta \varepsilon$ :

$$
\Delta \sigma^{\mathrm{t}}=\sum_{\mu=1}^{\mathrm{n}} \Delta \sigma_{\mu}^{\mathrm{t}}+\Delta \varepsilon \mathrm{E} 0=\sum_{\mu=1}^{\mathrm{n}}\left[\sigma_{\text {hist } \mu}^{\mathrm{t}}(\mathrm{t})+\frac{\Delta \varepsilon \tilde{\mathrm{E}}_{\mu}}{\Delta \mathrm{t}}\right]+\Delta \varepsilon \mathrm{E} 0
$$

avec : $\left\{\begin{array}{l}\tilde{\mathrm{E}}_{\mu}=\tau_{\mu} \mathrm{E}_{\mu}\left(1-\exp \left(\frac{-\Delta \mathrm{t}}{\tau_{\mu}}\right)\right) \\ \sigma_{\text {hist } \mu}^{\mathrm{t}}(\mathrm{t})=\sigma_{\mu}^{\mathrm{t}}(\mathrm{t})\left(\exp \left(\frac{-\Delta \mathrm{t}}{\tau_{\mu}}\right)-1\right)\end{array}\right.$

$\sigma_{\mu}^{t}(t)$ est la contrainte effective dans la branche $\mu$ de la chaîne de Maxwell à l'instant $t$. Une fois la contrainte effective évaluée, les équations [4] à [9] permettent de calculer l'endommagement, et donc la contrainte macroscopique appliquée au matériau (équation [20]). Nous utilisons la déformation totale appliquée à la chaîne de Maxwell dans les équations définissant la croissance de l'endommagement. 
L'intégration dans le temps, en chaque point de Gauss, est explicite à nouveau. Etant donné un incrément de déformation $\Delta \varepsilon$ au cours du pas de temps $\Delta t$, l'endommagement est directement calculé à partir des équations [5] à [9] et l'incrément de contrainte effective est calculé à partir des équations [21] et [22]. Nous pouvons alors calculer la raideur sécante de chaque élément à partir des équations [18] et [20].

Nous proposons dans la suite de calculer une variable que nous appelons $\varepsilon^{\text {in }}$ destinée à représenter une déformation instantanée fictive à partir de laquelle la « déformation de fluage » sera déduite.

$$
\begin{gathered}
\varepsilon^{\text {in }(t)}=\frac{\sigma(t)}{E(1-d)} \\
\varepsilon^{f l}(t)=\varepsilon(t)-\varepsilon^{\text {in }}(t) \\
\text { avec : } \quad E=\sum_{\mu=0}^{\mathrm{n}} E_{\mu}
\end{gathered}
$$

Contrairement au modèle de Kelvin, cette loi de comportement ne requiert pas le stockage de l'histoire de chargement. Il suffit de mettre à jour à chaque pas de temps les contraintes dans chaque branche de la chaîne de Maxwell. Celles-ci servent au calcul de l'incrément de contrainte au pas suivant.

Cette différence, assez fondamentale sur le plan numérique, fait que le modèle de Maxwell a souvent été préféré au modèle de Kelvin. En viscoélasticité linéaire, les deux approches sont équivalentes et la simplicité d'implantation numérique prime. Couplés à l'endommagement de la façon indiquée dans ce paragraphe et le précédent, les deux modèles rhéologiques ne sont plus du tout équivalents. Bien entendu, il est toujours possible d'utiliser une chaîne de Kelvin au lieu d'une chaîne de Maxwell pour calculer la contrainte effective mais à nouveau, l'implantation numérique plus complexe du modèle de Kelvin ne serait pas justifiée au regard de l'équivalence entre les deux représentations rhéologiques.

\section{Comparaisons en traction et en flexion}

Dans cette partie, nous comparons les deux approches du couplage endommagement-fluage présentées ci-dessus. Nous commencerons par l'exemple simple du fluage en traction d'un barreau et nous aborderons ensuite la relaxation de poutres sollicitées en flexion trois points.

\subsection{Simulations de fluage unidimensionnel}

Nous considérons pour cet exemple un barreau d'épaisseur $4 \mathrm{~cm}$, de hauteur $16 \mathrm{~cm}$ et de longueur $32 \mathrm{~cm}$. Celui-ci est encastré d'un côté et sollicité en fluage. Le 
niveau initial de contrainte vaut $1,23 \mathrm{MPa}$, ce qui correspond à $52 \%$ de la contrainte maximale en traction. Nous nous intéressons à l'évolution de la déformation dans le barreau (ou du déplacement à son extrémité). Nous considérons un maillage constitué d'un seul élément. Les paramètres du modèle d'endommagement considérés dans cet exemple sont donnés dans le tableau 1. Les différents paramètres de la chaîne de Maxwell sont donnés dans le tableau 3, ceux de la chaîne de Kelvin font l'objet du tableau 4. Les figures 3 et 4 montrent les déformations de fluage induites dans le barreau suite à la sollicitation en fluage.

\begin{tabular}{|c|c|c|c|c|c|}
\hline $\mathrm{E}(\mathrm{MPa})$ & $\mathrm{Ft}_{28}(\mathrm{MPa})$ & $\mathrm{A}_{\mathrm{t}}$ & $\mathrm{B}_{\mathrm{t}}$ & $\kappa_{0}$ & $1_{\mathrm{c}}$ \\
\hline 32700 & 2,4 & 1 & 600 & $1,5 \mathrm{E}-4$ & 0,08 \\
\hline
\end{tabular}

Tableau 1. Paramètres du modèle d'endommagement

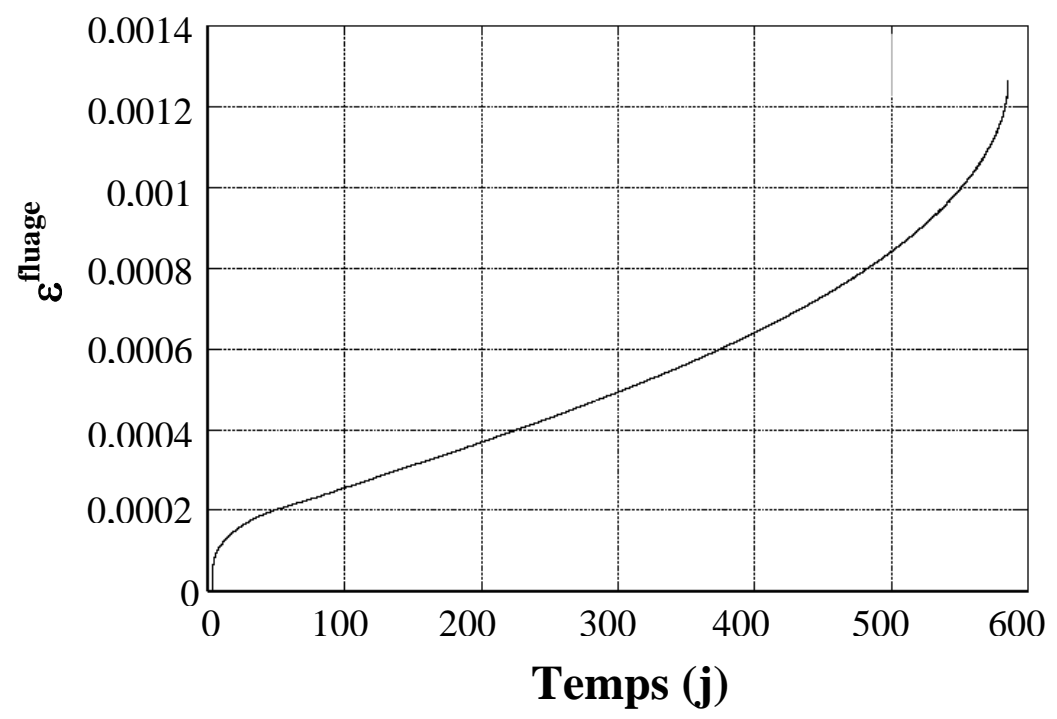

Figure 3. Réponse d'un barreau en traction sollicité en fluage avec le modèle de Maxwell

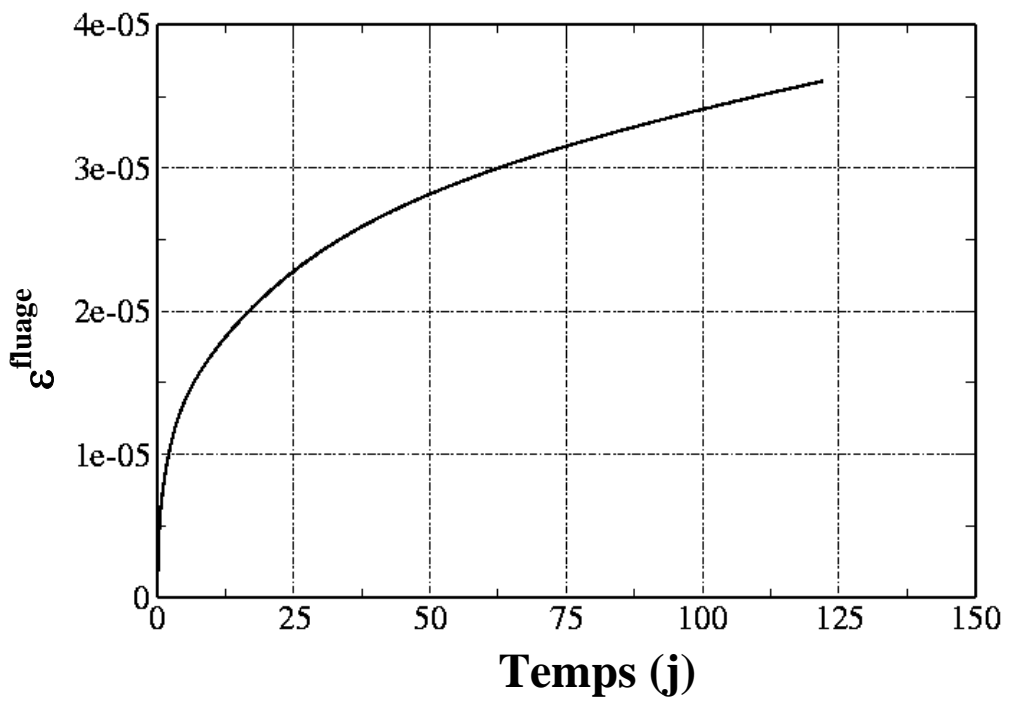

Figure 4. Réponse d'un barreau en traction sollicité en fluage avec le modèle de Kelvin 
Pour le modèle de Kelvin (sans endommagement), la réponse est triviale car elle correspond à la fonctionnelle de fluage introduite dans le modèle. Selon le niveau de contrainte appliqué, un endommagement apparaît ou non et reste constant au cours du temps, car la partie instantanée de la déformation est constante. La déformation du barreau est égale à la déformation de l'élément endommageable dans le modèle rhéologique, plus la déformation de fluage résultant de la fonctionnelle équation [11]. Ainsi, la vitesse de déformation du barreau a tendance à diminuer au court du temps. Nous nous sommes contentés d'une étude sur 125 jours, vu la complexité du calcul due au stockage de l'histoire de chargement dans ce modèle.

Pour le modèle de Maxwell, la réponse est différente. Initialement, la déformation est égale à celle des ressorts dans le modèle rhéologique. Au cours du temps, celle-ci va augmenter et l'endommagement va croître au-delà du seuil de déformation donné équations [4] et [7]. Ainsi, nous observerons une rupture du barreau sollicité par une charge constante au cours du temps.

Cette partie de la déformation de fluage, correspondant à la rupture du barreau, est appelée «fluage tertiaire». La déformation, la vitesse de déformation et l'endommagement augmentent, ce qui conduit à la rupture au sein du matériau.

Cette rupture possible constitue une différence fondamentale entre les deux modèles présentés. Notons que l'existence d'une rupture en fluage a été historiquement la motivation même de l'introduction d'une variable d'endommagement et du concept de contrainte effective (voir par exemple (Lemaitre, 1992).

\subsection{Relaxation en flexion}

Dans cette partie, nous considérons la relaxation de poutres en flexion trois points de portée $70 \mathrm{~cm}$, de hauteur $20 \mathrm{~cm}$ et d'épaisseur $10 \mathrm{~cm}$. Les maillages employés sont constitués de 16 éléments, chacun étant discrétisé en huit couches d'épaisseur égale.

Les paramètres de la loi d'endommagement sont calibrés selon une procédure d'identification basée sur des essais expérimentaux effectués sur un béton ordinaire (Omar, 2003). Ces paramètres sont donnés dans le tableau 2. En ce qui concerne la partie viscoélastique du comportement, nous avons utilisé une chaîne de Maxwell de quatre branches avec une branche de Hooke en parallèle entre eux et une chaîne de Kelvin avec huit éléments.

\begin{tabular}{|c|c|c|c|c|c|}
\hline $\mathrm{E}(\mathrm{MPa})$ & $\mathrm{Ft}_{28}(\mathrm{MPa})$ & $\mathrm{A}_{\mathrm{t}}$ & $\mathrm{B}_{\mathrm{t}}$ & $\kappa_{0}$ & $1_{\mathrm{c}}$ \\
\hline 39000 & 3,48 & 0,81175 & 25748 & $5,0^{\mathrm{E}}-05$ & 0,075 \\
\hline
\end{tabular}

Tableau 2. Paramètres de la loi d'endommagement mécanique 
Pour le modèle de Maxwell, les temps de relaxation de chaque branche ont été choisis à l'avance selon une progression géométrique proposée par Bazant (Bazant, 1974, les modules d'élasticité ont été ensuite calés sur la fonction de fluage donnée dans (Granger, 1995) pour des données numériques jusqu'à 1000 jours, la méthode de calage est donnée dans (Omar, 2001). Le tableau 3 résume les valeurs trouvées après calage.

\begin{tabular}{|l|l|}
\cline { 2 - 2 } \multicolumn{1}{c|}{} & $\mathrm{E}_{0}=15550 \mathrm{MPa}$ \\
\hline$\tau_{1}=1$ jour & $\mathrm{E}_{1}=7921 \mathrm{MPa}$ \\
\hline$\tau_{2}=10$ jours & $\mathrm{E}_{2}=4246 \mathrm{MPa}$ \\
\hline$\tau_{3}=100$ jours & $\mathrm{E}_{3}=5574 \mathrm{MPa}$ \\
\hline$\tau_{4}=1000$ jours & $\mathrm{E}_{4}=5061 \mathrm{MPa}$ \\
\hline
\end{tabular}

Tableau 3. Valeurs des paramètres de la chaîne de Maxwell pour $t_{0}=28$ jours

Le tableau 4 donne les paramètres correspondants à la chaîne de Kelvin.

\begin{tabular}{|l|l|}
\cline { 2 - 2 } \multicolumn{1}{c|}{} & $\mathrm{E}_{0}=33170 \mathrm{MPa}$ \\
\hline$\tau_{1}=0,002$ jours & $\mathrm{E}_{1}=786100 \mathrm{MPa}$ \\
\hline$\tau_{2}=0,02$ jours & $\mathrm{E}_{2}=816760 \mathrm{MPa}$ \\
\hline$\tau_{3}=0,2$ jours & $\mathrm{E}_{3}=346900 \mathrm{MPa}$ \\
\hline$\tau_{4}=2$ jours & $\mathrm{E}_{4}=183070 \mathrm{MPa}$ \\
\hline$\tau_{1}=20$ jour & $\mathrm{E}_{5}=102280 \mathrm{MPa}$ \\
\hline$\tau_{2}=200$ jours & $\mathrm{E}_{6}=52950 \mathrm{MPa}$ \\
\hline$\tau_{3}=2000$ jours & $\mathrm{E}_{7}=36100 \mathrm{MPa}$ \\
\hline$\tau_{4}=20000$ jours & $\mathrm{E}_{8}=17520 \mathrm{MPa}$ \\
\hline
\end{tabular}

Tableau 4. Valeurs des paramètres pour la chaîne de Kelvin pour $t_{0}=28$ jours

Nous analysons, dans un premier temps, l'influence du niveau de sollicitation initial sur la relaxation de cette poutre. Les chargements appliqués sont des déplacements constants qui correspondent à $85 \%$ de l'effort au pic. Le premier niveau est situé avant d'atteindre la charge maximale, le second est situé dans la phase adoucissante de la réponse de la poutre. Nous pouvons dessiner les courbes de relaxation $\mathrm{P}(\mathrm{t}) / \mathrm{P}_{0}$ où $\mathrm{P}_{0}$ est l'effort correspondant au début de la relaxation. La figure 5 montre les résultats obtenus avec le modèle de Maxwell couplé à l'endommagement. La courbe de relaxation correspondant à un chargement appliqué en post-pic vient en dessous de celle en pre-pic. Notons qu'avec ce modèle l'endommagement n'évolue pas lors de l'application d'un chargement de relaxation, il reste constant pendant toute 
la période du chargement. Ces résultats sont en accord qualitatif avec ceux de Gettu et Bazant (Gettu, 1992) qui consistaient à charger des poutres en flexion 3 points avec une vitesse de chargement constante, le déplacement imposé était ensuite maintenu constant à $85 \%$ de l'effort au pic en pre-pic pour le premier type d'essais, et en postpic pour le deuxième type. Ils ont montré que la relaxation est d'autant plus importante quand elle est imposée en post-pic.

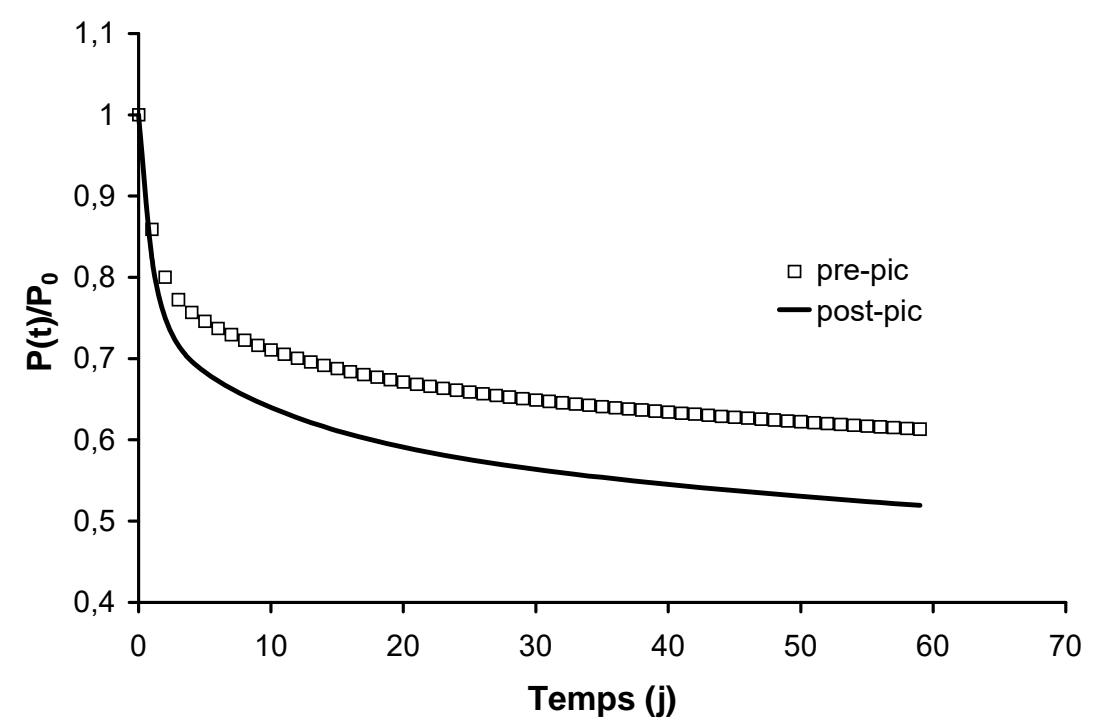

Figure 5. Courbes de relaxations $P(t) / P_{0}-$ modèle de Maxwell

La figure 6 présente les résultats obtenus avec le modèle de Kelvin. Comparés à ceux de la figure 5, ces résultats révèlent une contradiction non seulement avec le modèle précédent mais aussi par rapport à ce que nous avons trouvé dans la littérature (Gettu, 1992). Avec ce modèle, l'endommagement évolue avec le temps lors de l'application d'un chargement de relaxation. Le module sécant calculé en fonction de l'endommagement évolue avec un taux de dégradation plus important lors d'un chargement appliqué avant le pic que lors d'un chargement correspondant à la phase adoucissante.

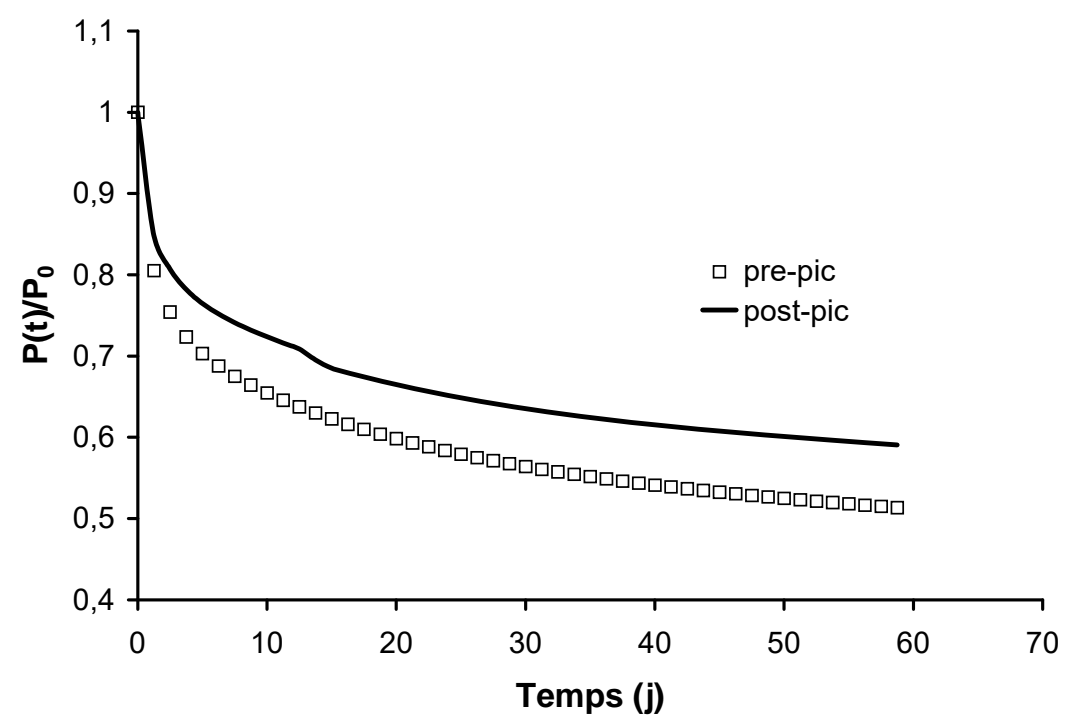

Figure 6. Courbes de relaxations $P(t) / P_{0}-$ modèle de Kelvin 
Dans les simulations suivantes, nous étudions l'influence de la longueur caractéristique sur la relaxation et sur la cinétique de fluage. Des calculs ont été conduits sur des poutres en flexion 3 points utilisant diverses valeurs de lc $(0,075 \mathrm{~m} ; 0,085 \mathrm{~m} ; 0,095 \mathrm{~m})$. Les figures 7 et 8 présentent les résultats obtenus avec le modèle de Maxwell couplé à l'endommagement pour un chargement de relaxation appliqué au milieu de la poutre et qui correspond à $85 \%$ de l'effort au pic en post-pic $(\mathrm{U}=-35,8 \mu \mathrm{m})$. Nous remarquons que l'effort augmente avec la longueur caractéristique du matériau, par ailleurs la déformation de fluage est fortement influencée par lc. Elle est d'autant plus petite que la longueur interne est plus grande. Lorsque lc augmente, la taille de la Fracture Process Zone est plus grande, ce qui implique une augmentation relative de la contrainte (en négligeant les effets de singularité en pointe de fissure), et par la suite un effort plus grand et une déformation de fluage plus petite.

Concernant les résultats par le modèle de Kelvin couplé à l'endommagement, les figures 9 et 10 montrent que l'effort augmente aussi avec lc mais, contrairement au modèle de Maxwell, la déformation de fluage se trouve réduite lorsque lc augmente.

Avec ces deux modèles lorsque lc augmente l'effort augmente. Dans le cas du modèle de Maxwell, l'état de déformation est constant dans la poutre, la déformation instantanée $\varepsilon^{\text {in }}$ (équation [23]) est proportionnelle à la contrainte, la déformation de fluage diminue avec l'augmentation de la déformation instantanée et donc avec l'augmentation de la contrainte.

Dans le modèle de Kelvin, l'incrément de déformation de fluage est directement calculé à partir de la contrainte appliquée, ce qui explique l'augmentation de la déformation de fluage lorsque la contrainte (l'effort) augmente.

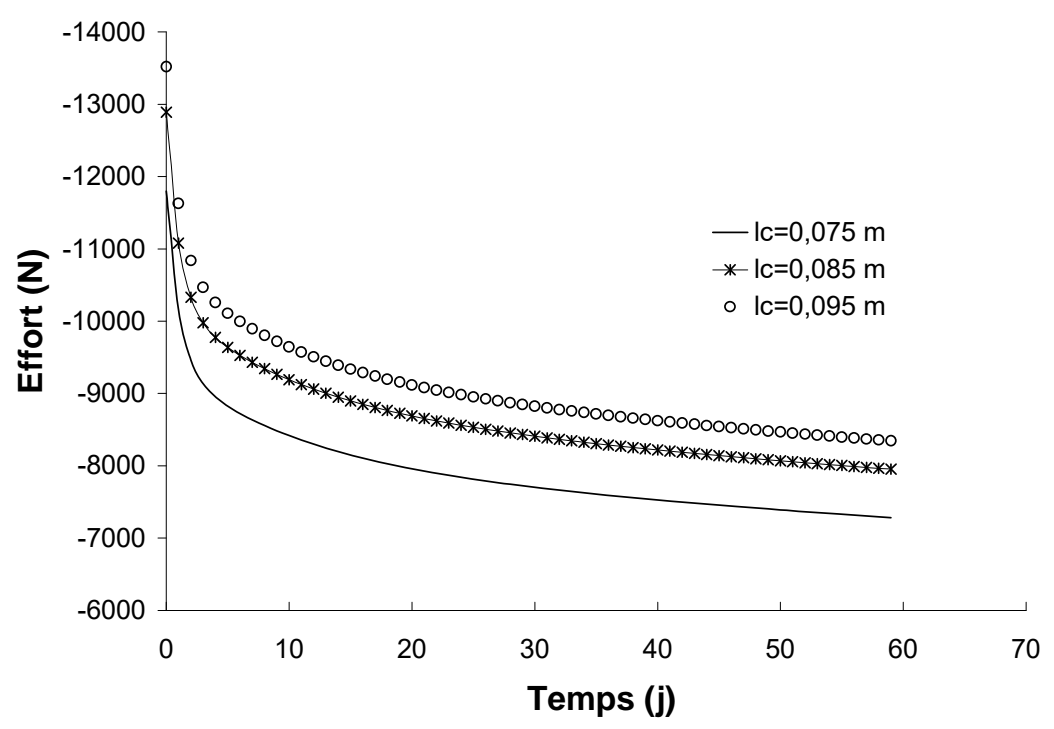

Figure 7. Effort-temps, influence de lc-modèle de Maxwell 


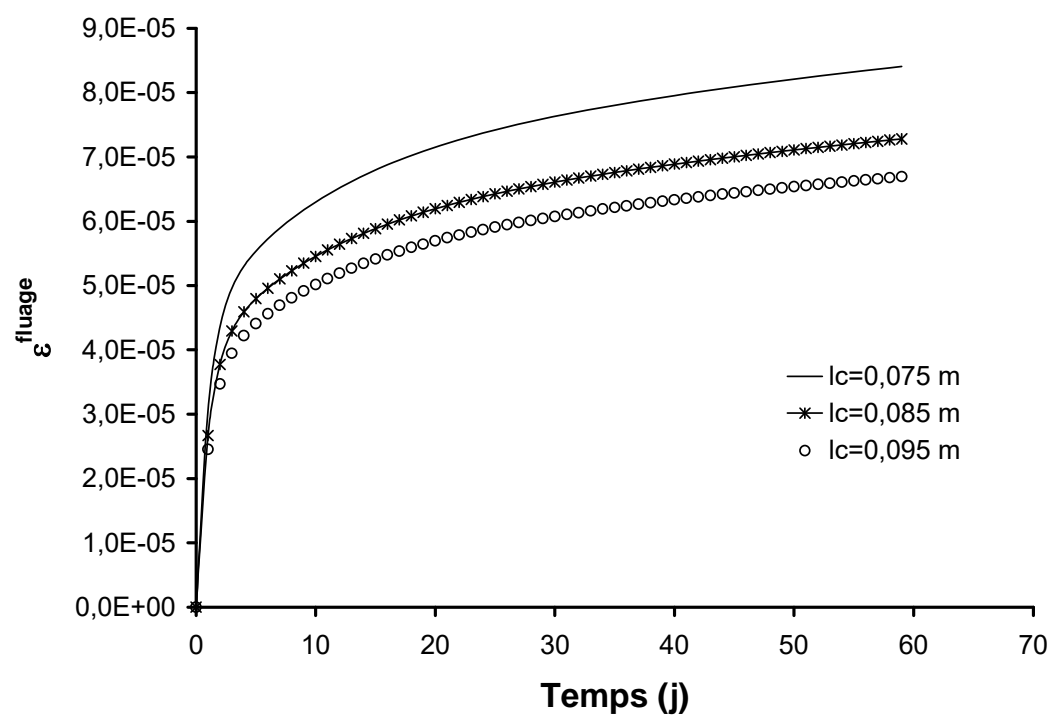

Figure 8. $\varepsilon^{\text {fluage }} /$ emps, influence de lc-modèle de Maxwell

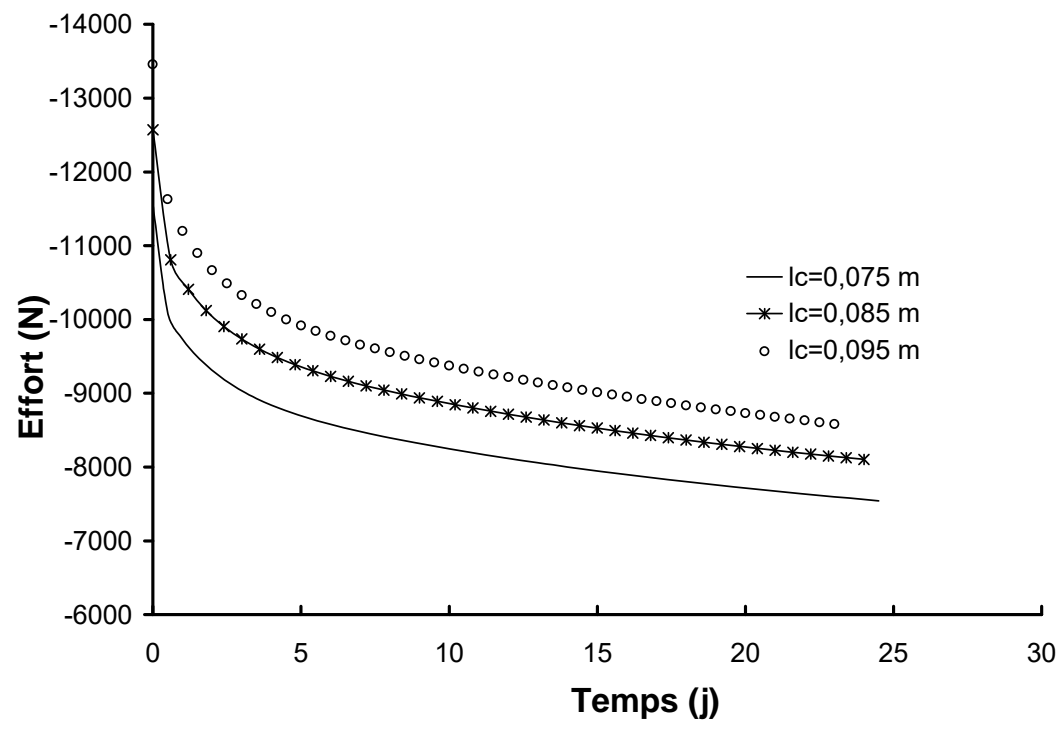

Figure 9. Effort/temps, influence de lc-modèle de Kelvin

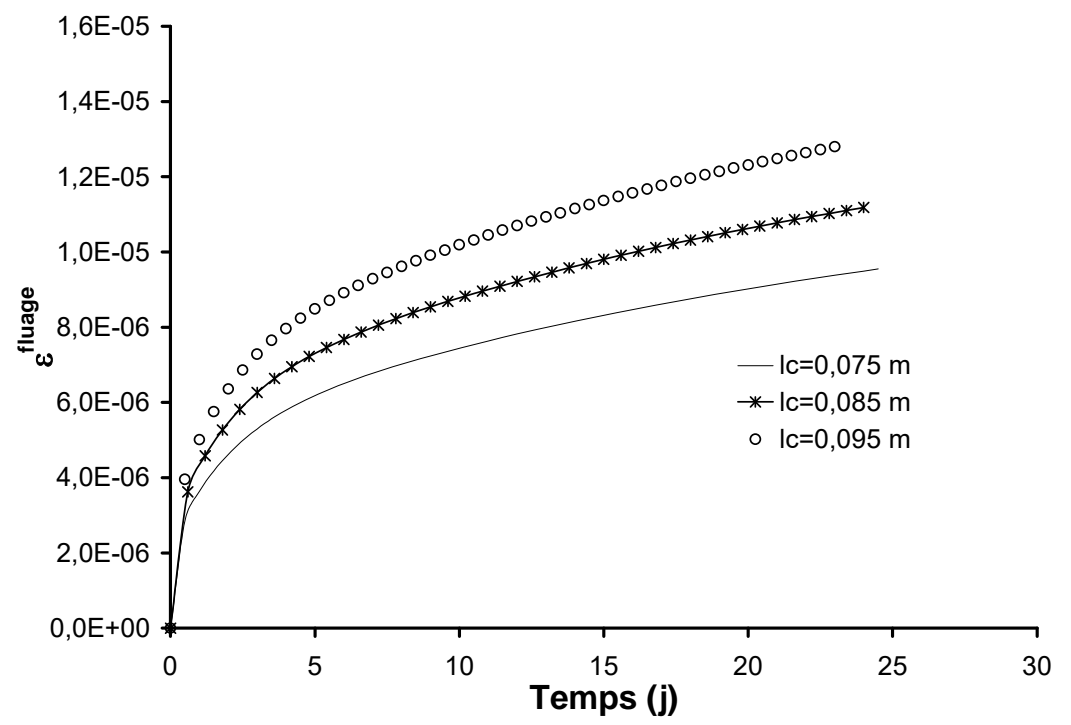

Figure 10. $\varepsilon^{\text {fluage } / t e m p s, ~ i n f l u e n c e ~ d e ~ l c-m o d e ̀ l e ~ d e ~ K e l v i n ~}$ 
Ces simulations permettent aussi de comparer, d'une part, la réponse en fluage de la fibre la plus comprimée et celle de la fibre la plus tendue. Pour un calcul avec le modèle de Maxwell couplé à l'endommagement, nous observons que la fibre tendue flue d'avantage que la fibre comprimée (figure 11), alors que le modèle couplé endommagement-chaîne de Kelvin aboutit à l'inverse dans les mêmes conditions de maillage et de chargement (figure 12).

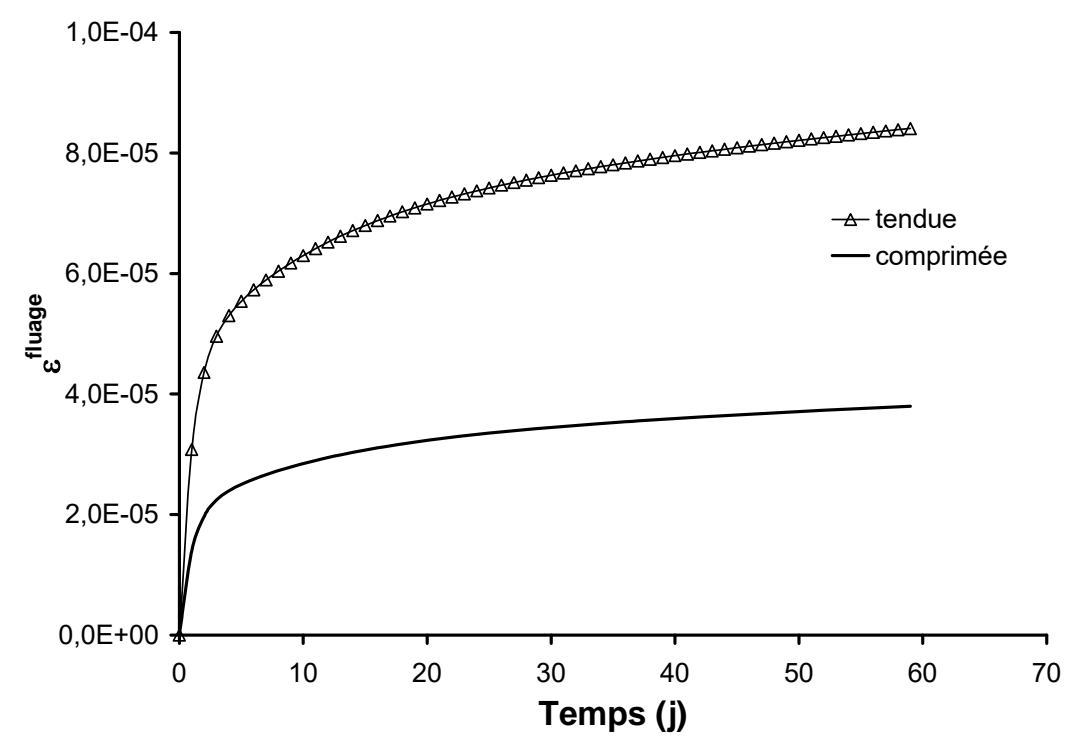

Figure 11. $\varepsilon^{\text {fluage} / t e m p s, ~ f i b r e ~ t e n d u e ~-~ f i b r e ~ c o m p r i m e ́ e ~(c h a i ̂ n e ~ d e ~ M a x w e l l) ~}$

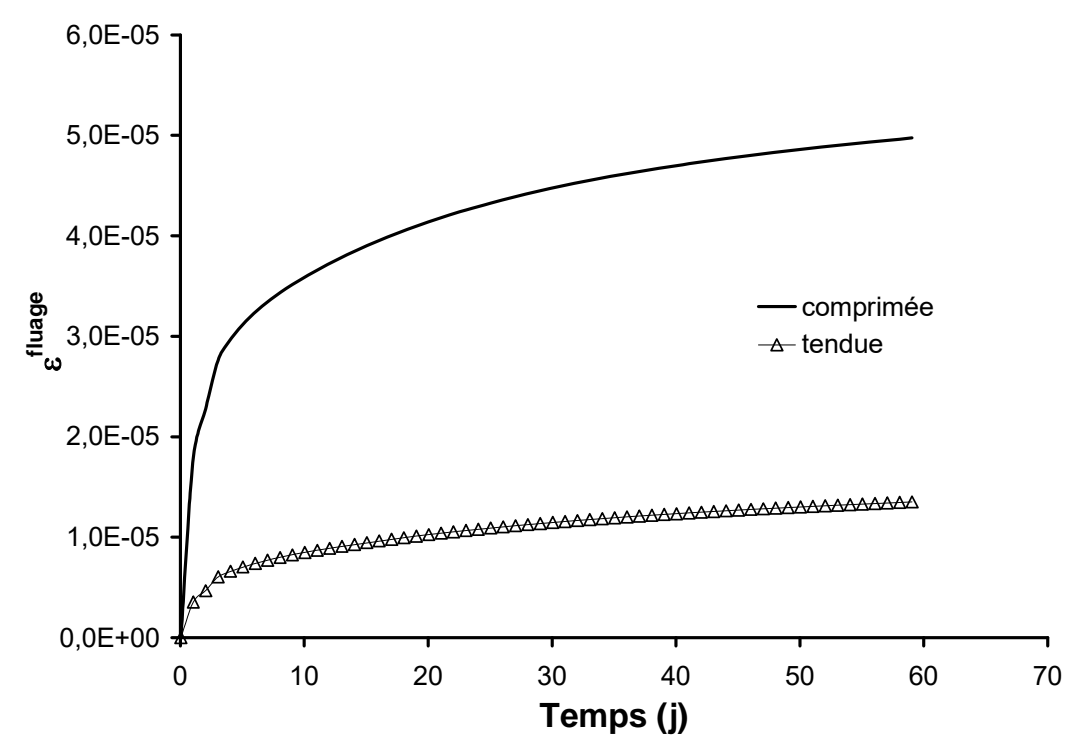

Figure 12. $\varepsilon^{\text {fluage }} /$ temps, fibre tendue - fibre comprimée (chaîne de Kelvin)

Nous constatons qu'entre les deux modèles de couplage, seul le modèle de Maxwell couplé à l'endommagement via la contrainte effective est capable de décrire la rupture des poutres lors d'un essai de fluage. Il permet aussi de prédire au moins qualitativement les résultats d'essais de relaxation des poutres en flexion obtenus dans les essais de Gettu et Bazant (Gettu, 1992). 


\section{Etudes complémentaires sur le modèle de Maxwell}

\subsection{Localisation de l'endommagement}

Dans ce paragraphe nous considérons le problème unidimensionnel d'une barre en traction dont les caractéristiques sont présentées dans la figure 13. Afin de limiter la localisation de l'endommagement dans une zone bien définie à l'avance, nous créons au centre de la barre un défaut initial. Cela consiste à introduire un élément dont la rigidité initiale est $10 \%$ plus faible que la rigidité des autres éléments du maillage. La barre a une longueur $60 \mathrm{~cm}$, une hauteur de $20 \mathrm{~cm}$, une épaisseur de $10 \mathrm{~cm}$, et la valeur choisie de la longueur interne est de $12 \mathrm{~cm}$. Le maillage éléments finis est constitué de 40 éléments de dimensions égales (inférieures à la longueur interne du matériau). Les paramètres du matériau utilisé pour cette étude sont reportés dans les tableaux 2 et 3.

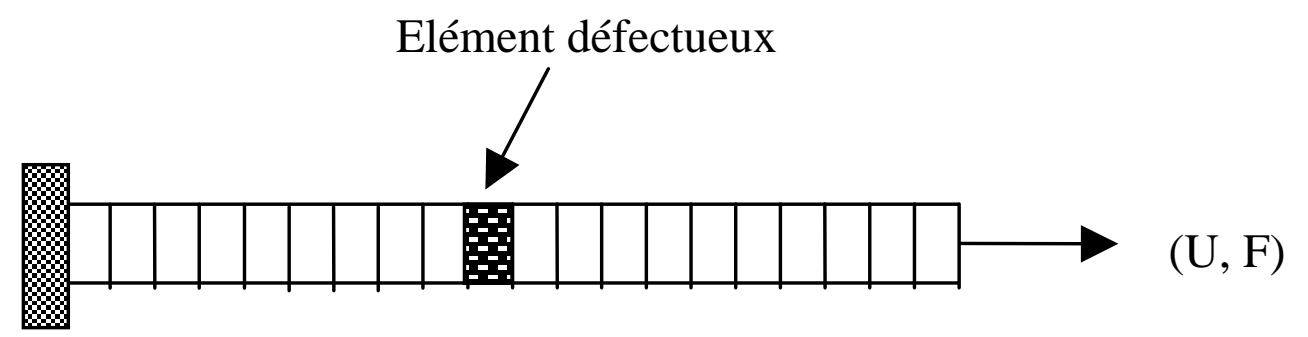

Figure 13. Barre unidimensionnelle chargée en traction

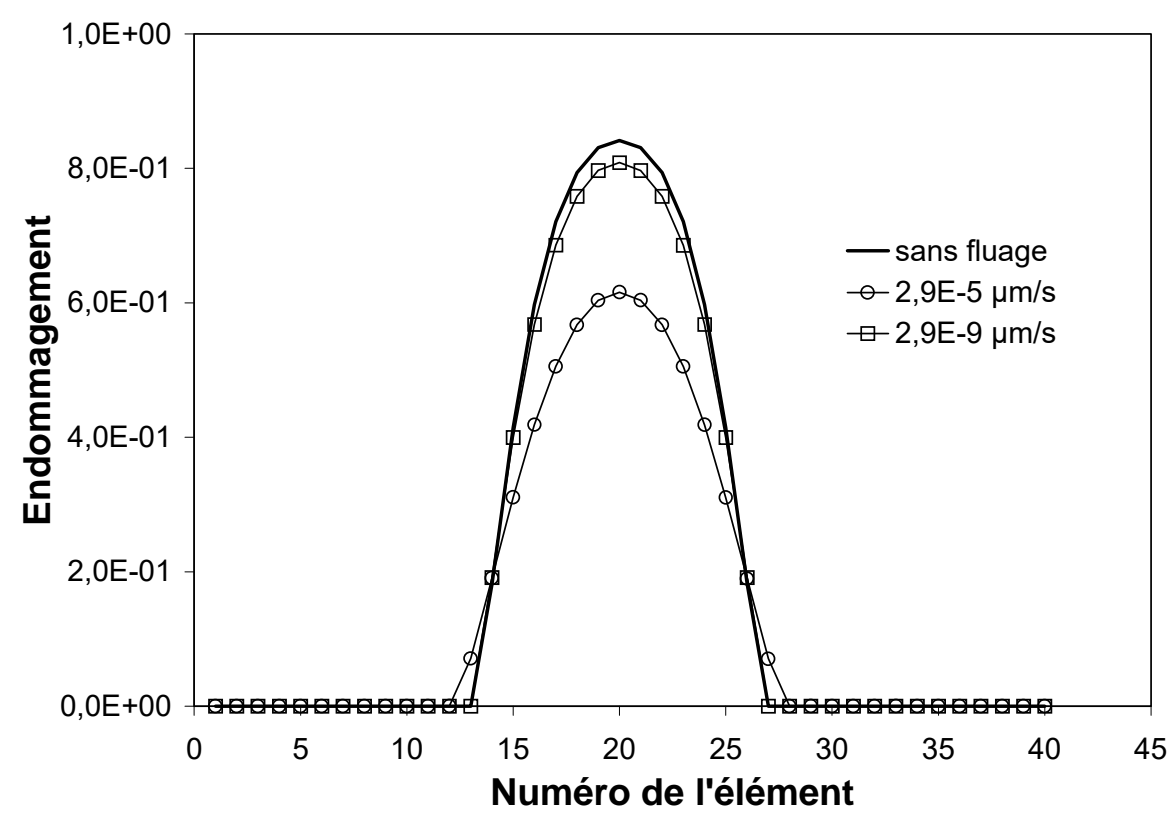

Figure 14. Evolution des profils d'endommagement avec la vitesse de chargement au même instant de chargement 
La figure 14 montre l'évolution des profils d'endommagement avec la vitesse de chargement. Nous remarquons que la vitesse de chargement n'influe pas sur la zone de localisation de l'endommagement. Il est délicat de comparer les cinétiques d'endommagement entre elles, surtout pour le modèle d'endommagement élastique (sans viscoélasticité). On observe cependant que cette cinétique est accélérée lorsque la vitesse de chargement est plus lente.

L'augmentation de la fragilité se traduit par une diminution de la longueur caractéristique du matériau (Bazant, 1989), cette fragilité est influencée par la vitesse de chargement. D'autre part, Haidar a démontré que la longueur caractéristique n'est pas constante mais elle évolue en fonction du niveau de chargement (Haidar, 2002). Ses résultats ont été justifiés qualitativement à travers des essais expérimentaux d'émission acoustique. Actuellement notre modèle numérique n'est pas capable de représenter cette variation de la longueur interne en fonction de la vitesse de chargement.

\subsection{Effet de la vitesse de chargement}

Un autre paramètre aussi important que la longueur interne du matériau est la vitesse avec laquelle on atteint la charge maximale lors d'un essai de flexion 3 points. Plusieurs auteurs ont évoqué l'importance de ce paramètre, parmi lesquels Gettu et Bazant qui ont démontré que la vitesse de chargement exerce un grand effet sur le comportement à la rupture des bétons (Gettu, 1992).

La figure 15 considère le cas d'un chargement monotone pour lequel nous étudions la sensibilité du comportement de la poutre à la vitesse de chargement. Dans ce cas, le corps d'épreuve est soumis à un déplacement appliqué à son milieu et qui croît avec un taux constant jusqu'à la rupture complète. Nous remarquons que la capacité de la poutre décroît avec la vitesse de chargement. Pc est la charge maximale correspondant à la vitesse de chargement la plus lente, $\mathrm{P}$ représente la charge calculée. La figure 15a montre une augmentation de $20 \%$ du pic de force pour une vitesse de $1,66 \times 10^{-4} \mu \mathrm{m} / \mathrm{s}$ qui correspond à dix fois la vitesse de chargement la plus lente $\left(\mathrm{v}=1,66 \times 10^{-5}\right)$, et de $40 \%$ pour une vitesse de $1,66 \mu \mathrm{m} / \mathrm{s}$.

On observe que le modèle de Maxwell couplé à l'endommagement reproduit d'une manière qualitative les résultats observés dans la littérature (figure 15b) (Wu, 1993). Cependant, il existe un niveau de vitesse de chargement à partir duquel cette influence devient médiocre et les réponses effort-flèche pour les différentes vitesses concernées s'approchent de la réponse mécanique sans fluage. Pour des vitesses plus grandes il faut un modèle d'endommagement visqueux (Dubé, 1996). Dans le cas présenté, le seuil critique de vitesse à partir duquel il n'y a plus de différence est de l'ordre de $10^{-2} \mu \mathrm{m} / \mathrm{s}$. 


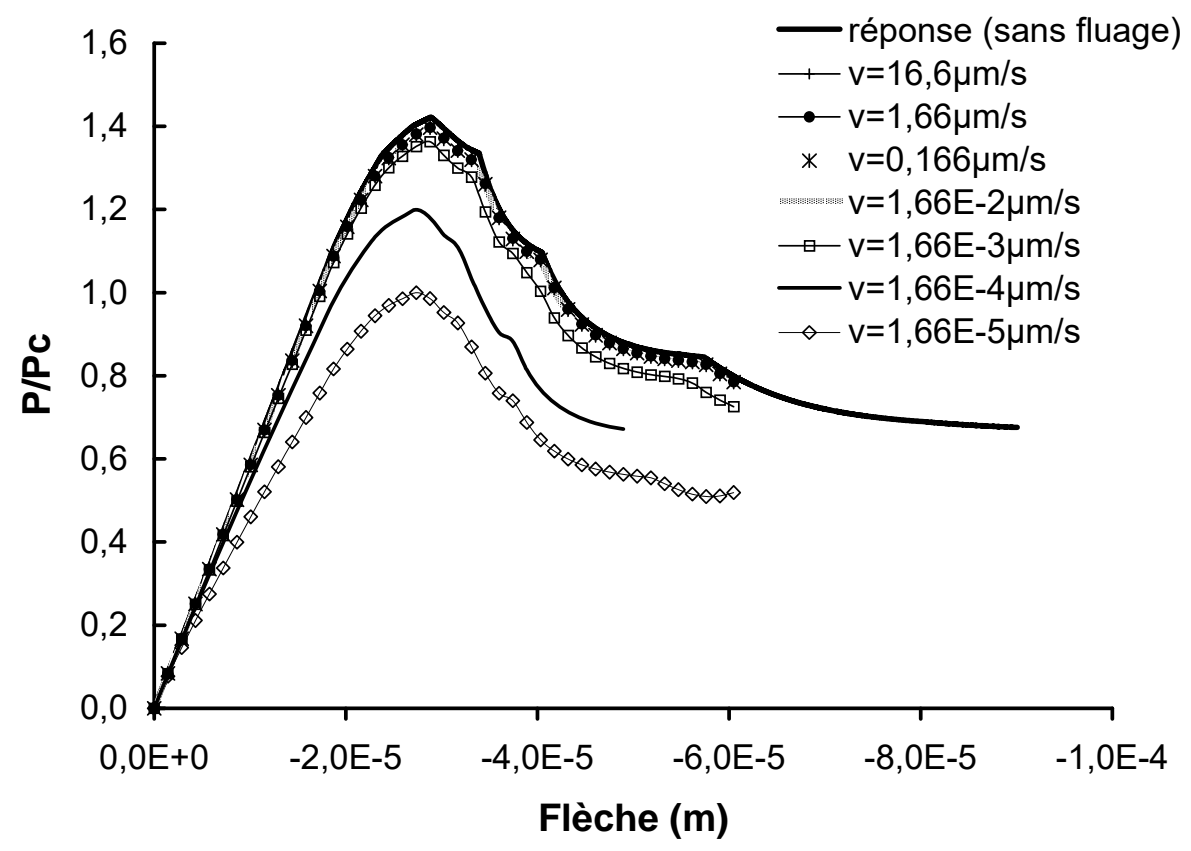

Figure 15a. Effort-flèche, influence de la vitesse de chargement

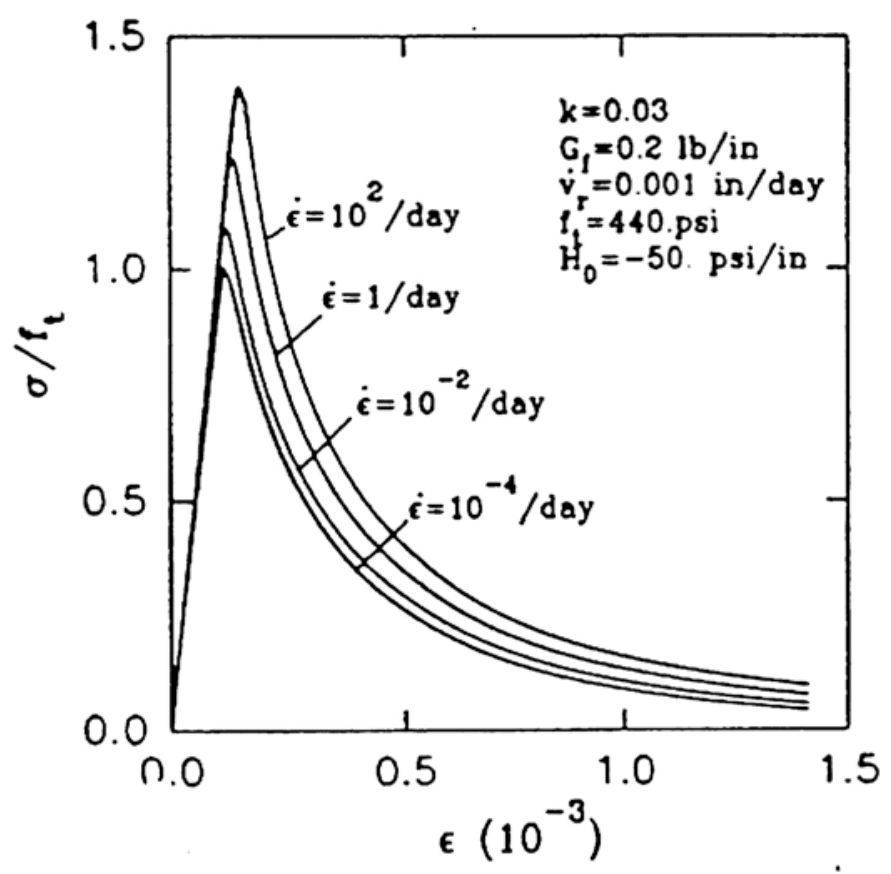

Figure 15b. Contrainte-déformation, influence de la vitesse de chargement d'après (Wu, 1993)

\subsection{Influence de la relaxation sur la résistance résiduelle}

Les simulations numériques suivantes consistent à solliciter, dans un premier temps, en relaxation des poutres en flexion, leurs résistances résiduelles à la flexion 
seront ensuite mesurées. Ce type d'étude correspond aux essais expérimentaux que nous menons en parallèle de cette étude numérique et qui permettront de dégager des données expérimentales destinées à évaluer la pertinence des modèles numériques. L'histoire de chargement est comme suit : un déplacement constant est imposé au milieu de la poutre pendant une certaine période de temps, il sera ensuite augmenté avec un taux de croissance constant jusqu'à la rupture des poutres.

La durée de relaxation influe significativement la résistance résiduelle des poutres. Dans le cas des simulations de la figure 16 , les poutres sont soumises à une relaxation dont le déplacement imposé correspond à $70 \%$ du déplacement au pic $(\mathrm{U}=-18,2 \mu \mathrm{m})$ de l'effort obtenu à partir de la réponse mécanique sans relaxation qui apparaît en trait gras. Ce chargement a été imposé pour trois différentes périodes de temps (10 jours, 90 jours et 120 jours). Les poutres sont ensuite soumises à un déplacement croissant jusqu'à sa rupture. La figure 16 montre en premier lieu que l'effort appliqué diminue sous l'effet de la relaxation, et en deuxième lieu que la résistance résiduelle, qui est une réponse à l'histoire de chargement évoqué ci-dessus, décroît en passant à une durée de relaxation plus grande.

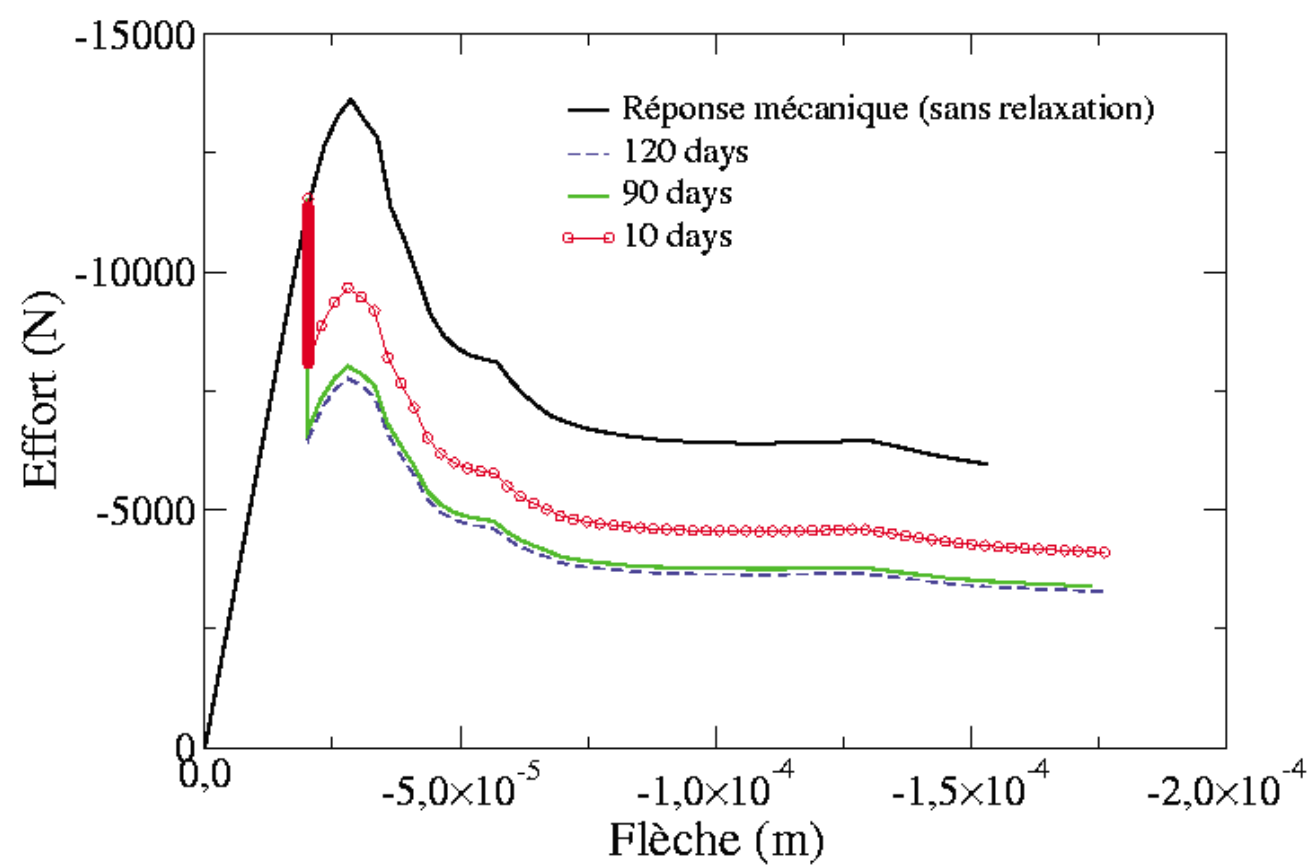

Figure 16. Courbe effort-flèche, influence de la durée de relaxation sur la résistance résiduelle

Comme pour les calculs faits au paragraphe 4.2, nous étudions dans ce paragraphe l'influence de la vitesse de chargement (figure 17). Nous visons des vitesses assez lentes pour lesquelles les propriétés de fluage commencent à jouer un rôle significatif dans la réponse de la structure. Nous considérons la même histoire de chargement que précédemment et une période de 10 jours de relaxation. Parmi les vitesses de chargement qui ont été considérées il y en a une qui correspond à la 
vitesse de chargement des essais expérimentaux que nous menons avec le même matériau et la même histoire de chargement $(\mathrm{v}=0,03 \mu \mathrm{m} / \mathrm{s})$.

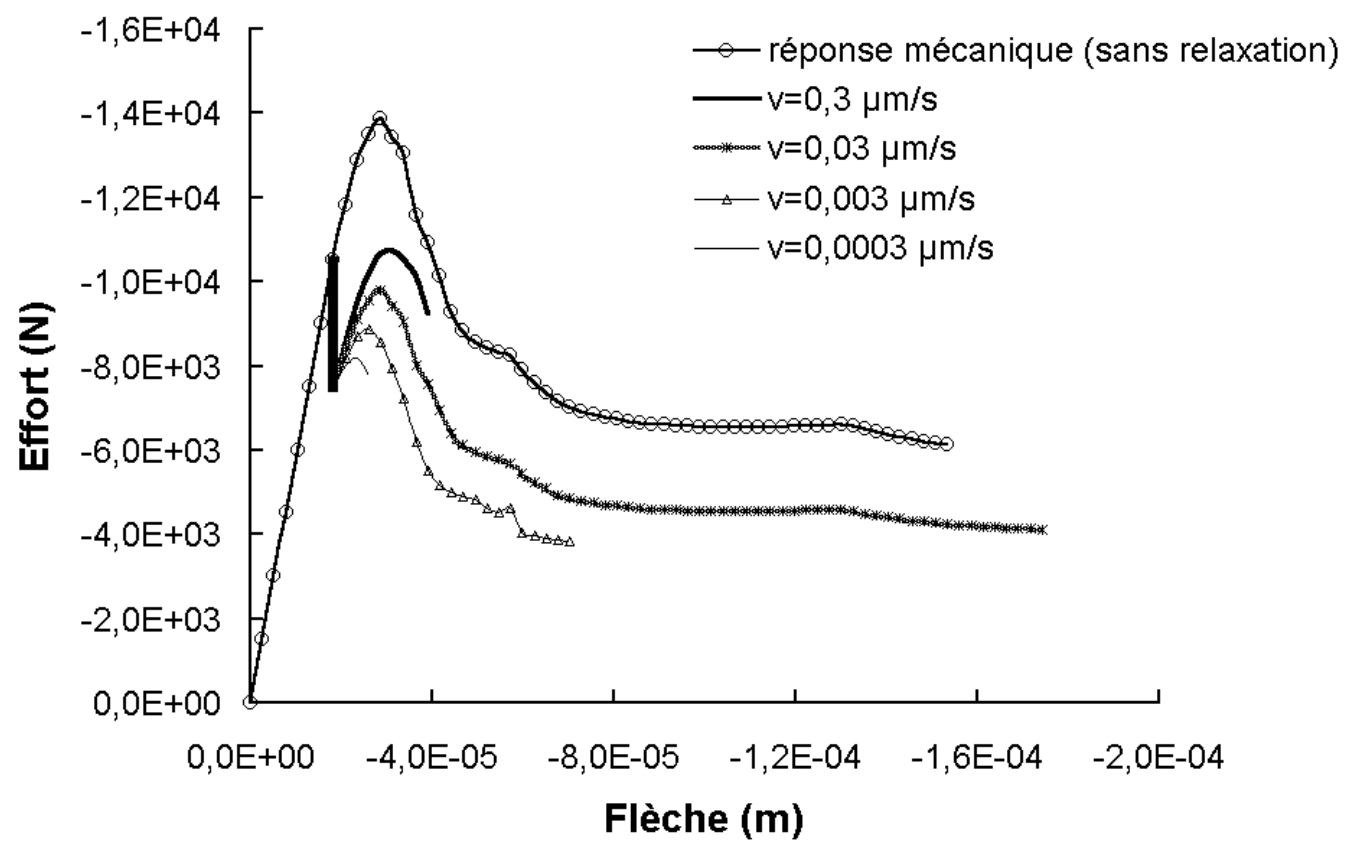

Figure 17. Effort-flèche, influence de la vitesse de chargement sur la résistance résiduelle

Nous remarquons à nouveau que l'effort maximal diminue avec la vitesse de chargement mais aussi qu'il est influencé par les effets de la relaxation et nous obtenons une résistance inférieure à celle du cas d'un chargement monotone. Ainsi en comparant sur la figure 17 la résistance résiduelle de la poutre relaxée avant la rupture par rapport à celle d'un chargement monotone nous remarquons que pour une vitesse $\mathrm{v}=0,03 \mu \mathrm{m} / \mathrm{s}$, l'effort maximal a diminué de l'ordre de $40 \%$. Les arrêts des courbes correspondant aux vitesses $0,3 \mu \mathrm{m} / \mathrm{s}$ et $0,0003 \mu \mathrm{m} / \mathrm{s}$ sont dus à une rupture de la poutre simulée.

L'influence de la longueur caractéristique sur la résistance résiduelle est donnée sur la figure 18 qui présente les courbes effort-flèche correspondant à chaque valeur de lc $(0,075 \mathrm{~m} ; 0,085 \mathrm{~m} ; 0,095 \mathrm{~m})$, l'essai de flexion 3 points est effectué pour les trois simulations avec une vitesse de chargement de $0,03 \mu \mathrm{m} / \mathrm{s}$. Nous observons que les résultats obtenus sont comparables à ceux que l'on trouve dans le modèle classique d'endommagement. En effet, la capacité résiduelle diminue avec lc. Ceci étant dit, soulignons l'importance de l'effet de la longueur caractéristique du matériau sur les prédictions du modèle, ce qui impose la nécessité d'une bonne détermination de ce paramètre ainsi qu'une connaissance de ses variations dues au fluage. Cet aspect fera l'objet d'une future campagne d'essais qui consiste à effectuer des essais d'émissions acoustiques sur des poutres qui ont subi un fluage. 


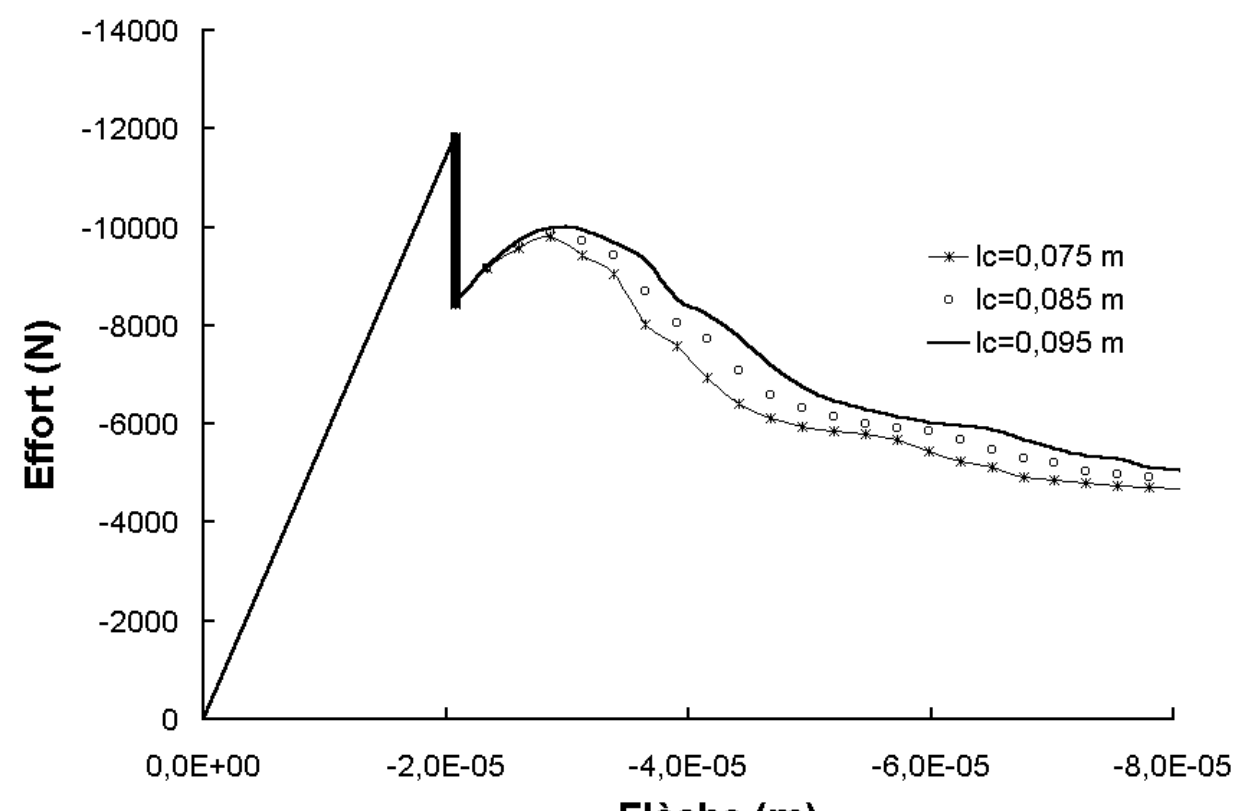

Flèche $(\mathrm{m})$

Figure 18. Effort-flèche, influence de la longueur interne sur la résistance résiduelle

\section{Conclusions}

Notre travail a pour objectif le développement d'outils numériques capables de prédire et de comprendre le comportement différé de structures, notamment le fluage et la relaxation qui s'avèrent être délicats puisqu'ils agissent, de façon plus ou moins couplés, avec d'autres phénomènes, dont la fissuration. Nous avons présenté deux techniques de couplages entre le comportement visqueux et le comportement endommageable du béton. Des modèles unidimensionnels ont été implantés dans un code de calcul d'ossatures sollicitées en traction et en flexion. Les différentes simulations effectuées mettent en évidence la contribution importante des effets d'endommagement sur le fluage et la relaxation mesurés dans le domaine de viscoélasticité associée à la rupture du béton. Ces simulations nous ont permis de dégager les conclusions suivantes :

- entre les deux techniques de couplages, seule la modélisation (couplage endommagement-chaîne de Maxwell) basée sur le concept de la contrainte effective, permet de décrire la croissance de l'endommagement lors d'un essai de fluage ;

- la cinétique de fluage est largement influencée par la longueur interne du matériau ; contrairement au modèle couplé endommagement-chaîne de Kelvin, avec le modèle de Maxwell, cette cinétique augmente pour une longueur interne plus petite ; les résultats obtenus avec ce modèle pour des poutres en flexion soumises à la relaxation sont cohérents avec les résultats publiés dans la litérature (Bazant, 1992). 
Notons qu'en utilisant le modèle de Kelvin couplé à l'endommagement, nous avons observé une convergence très lente des calculs lors de raffinements de maillages, il convient donc d'utiliser des stratégies de calcul mieux optimisées pour limiter ce phénomène, en évitant le stockage excessif de l'histoire de chargements qui encombre la mémoire, ce qui est le cas du modèle couplé endommagementchaîne de Maxwell. Une étude complémentaire sur ce modèle a permis de tirer les conclusions suivantes :

- dans le cas de poutres en flexion soumises à la relaxation suivie d'un chargement monotone jusqu'à la rupture, la résistance des poutres diminue avec la longueur interne; ces résultats sont comparables à ceux du modèle classique d'endommagement; la détermination de la longueur interne doit être faite avec précision, car elle conditionne grandement la qualité des prédictions ;

- la vitesse de chargement influe beaucoup sur le comportement des poutres en flexion 3 points ; les simulations sont en accord qualitatif avec les résultats observés dans la littérature (Wu, 1993); en effet, la capacité des poutres à la flexion diminue avec la vitesse de chargement; elle diminue aussi sous l'action de la relaxation; ainsi, en augmentant la durée de relaxation, la résistance résiduelle des poutres devient de plus en plus faible ;

- dans le cas d'une barre en traction présentant un défaut en son milieu, la zone de localisation de l'endommagement n'a pas été affectée par la variation de la vitesse de chargement.

Remerciements

Nous remercions le soutien financier de EDF-R\&D dans le cadre de son partenariat avec l'ERT R\&DO.

\section{Bibliographie}

Bazant Z.P., Wu S.T. "Dirichlet series creep function for ageing concrete", Journal of Engineering Mechanics Division ASCE 99 (Em2), p. 367-387, 1973.

Bazant Z.P., Wu S.T, "Rate type creep law of ageing concrete based on Maxwell chain", Materials and structures, vol. 7, n 37, p. 45-60, 1974.

Bazant Z.P., Pijaudier-Cabot G., Pan J-Y., "Strain-Softening in Reinforced Concrete Beams and Frames", Journal of Structural Engineering, ASCE, vol. 113, n 12, p. 2333-2347, 1987.

Bazant Z.P., Pijaudier-Cabot G., Pan J-Y., "Ductility, snapback, size effect and redistribution in softening beams and frames", ASCE J. of Structural Engng., 113, p. 2348-2364, 1987.

Bazant Z.P., Mathematical modeling of creep and shrinkage of concrete, John Wiley \& Sons Ltd, 1988. 
Bazant Z.P., Pijaudier-Cabot G., "Measurement of characteristic length of nonlocal continuum”, Journal of Engineering Mechanics. ASCE, 115, p. 755-767, 1989.

Bazant Z.P., Jirasek M., "R-curve modeling of rate effect in static fracture and its interference with size effect, in Fracture Mechanics of Concrete Structures", Proc. Int. Conf. On Fracture Mechanics of Concrete Structures. Breckenridge, Colorado, June, ed. by Bazant Z.P., Elsevier Applied Science, London, p. 918-923, 1992.

Bazant Z.P., Planas J., Fracture and size effect in concrete and other quasibrittle materials, CRC press, Boca Raton and London, 1998.

Benboudjema F., Meftah F., Sellier A., Heinfling G., Torrenti J.M., "A basic creep model for concrete subjected to multiaxial loads", Fracture Mechanics of Concrete Structures, édité par R. de Borst, J. Mazars, G. Pijaudier-Cabot et J.G.M. Van Mier, Swets \& Zeitlinger, Lisse, The Netherlands, p. 161-168, 2001.

Bissonnette B., Pigeon M., "Tensile creep at early ages of ordinary, Silica fume and fiber reinforced concretes", Cement and Concrete Research, vol. 25, n ${ }^{\circ}$ 5, p. 1075-1085, Elsevier Science Ltd, 1995.

De Borst R., Van den Boogaard A.H., Sluys L.J., "Computational issues in time-dependent deformations and fracture of concrete", Proc. The $5^{\text {th }}$ Int. RILEM Symposium on Creep and shrinkage of concrete, Barcelone, édité par Z.P. Bazant and I. Carol, p. 309-325, 1993.

Dubé J.F., Pijaudier-Cabot G., La Borderie C., "Rate dependent damage model for concrete dynamics", Journal of Engineering Mechanics, p. 939-947, October 1996.

Haidar K., Modélisation de l'endommagement des structures en béton - appoches numériques et effet de la microstructure sur les propriétés de rupture, Thèse de doctorat de l'Ecole Centrale de Nantes, 2002.

Gettu, R., Bazant, Z.P., "Rate effects and load relaxation: static fracture of concrete", ACI Materials J., 89 (5), p. 456-468, 1992.

Granger L., Comportement différé du béton dans les enceintes de centrales nucléaires (analyse et modélisation), Thèse de doctorat de l'ENPC, 1995.

Lemaitre J., Chaboche J.L., Mécanique des matériaux solide, Edition Dunod, 1984.

Lemaître J., A course on damage mechanics, Springer-Verlag, New York, Inc., 1992.

Mazars J., Application de la mécanique de l'endommagement au comportement non linéaire et à la rupture du béton de structures, Thèse de doctorat d'état, Université Paris-VI, 1984.

Mazzotti C., Savoia M., Tralli A., "An isotropic damage model for non linear creep behavior of concrete in compression", Fracture Mechanics of Concrete Structures, édité par R. de Borst, J. Mazars, G. Pijaudier-Cabot et J.G.M. Van Mier, Swets \& Zeitlinger, Lisse, The Netherlands, p. 255-262, 2001.

Omar M., Couplage déformations différées - fissuration du béton, DEA, Ecole Centrale de Nantes, 2000.

Omar M., Pijaudier-Cabot G., Modèle filaire simplifié, couplage fluage-endommagement, Rapport interne, EDF-R\&DO, 31 pages, 2001.

Omar M, Pijaudier-Cabot G., Loukili A., "Numerical models for coupling creep and fracture of concrete structures", Computational Modelling of Concrete Structures, Euro-C, St Johann, Austria, p. 531-539, 2003. 
Østergaard L., Lange D.A., Altoubat S.A., Stang H., "Tensile basic creep of early age concrete under constant load", Cement and Concrete Research, vol. 31, p. 1895-1899, Elsevier Science Ltd., 2001.

Pijaudier-Cabot G.,Bazant Z.P., "Nonlocal Damage Theory", J. of Engrg. Mech., ASCE, vol. 113, p. 1512-1533, 1987.

Pijaudier-Cabot G., Huerta A., "Finite Element Analysis of Bifurcation in Nonlocal Strain Softening Solids", Comp. Meth. in Applied Mech. and Engrg., vol. 90, p. 905-919, 1991.

Rots J.G., Computational modeling of concrete structures, Ph.D. Thesis, Delft University of Technology, Netherlands, 1988.

Rüsch H., Sell R., Rasch C., Stöckl S., "Investigations on the Strength of Concrete under Sustained Load", RILEM Symposium on the Influence of Time on the Strength and Deformation of concrete, Munich, 1958.

Santhikumar S., Karihaloo B.L., "Time-dependent tension softening", Mechanics of Cohesive-Frictional Materials, vol. 1, p. 295-304, 1996.

Weiss W.J., Prediction of early-age shrinkage cracking in concrete, PH.D. dissertation, Northwestern University, Evanston, Illinois, 1999.

Witasse R., Un modèle numérique pour l'étude du comportement hydro-mécanique du béton: application sur structure en béton armé soumise au fluage de dessiccation, Concours jeunes chercheurs 2000 - Prix René Houpert, p. 415-422, $27-28$ juin 2000.

Wu Z.S., Bazant Z.P., "Finite element modeling of rate effect in concrete fracture with influence of creep", Proc., $5^{\text {th }}$ int. Conf., CONCREEP-5, Barcelone, édité par Z.P. Bazant and I. Carol, p. 427-432, 1993.

Zi G., Bazant Z.P, "Continuous relaxation spectrum of concrete creep and its incorporation incorporation into microplane model M4", Proc., $6^{\text {th }}$ int. Conf., CONCREEP-6, MIT, Cambridge, Elsevier, p. 239-243, 2001. 